This is an electronic reprint of the original article. This reprint may differ from the original in pagination and typographic detail.

Author(s): Mattila, Keijo; Philippi, Paulo C.; Hegele, Luiz A.

Title: High-order regularization in lattice-Boltzmann equations

Year: $\quad 2017$

Version:

Please cite the original version:

Mattila, K., Philippi, P. C., \& Hegele, L. A. (2017). High-order regularization in latticeBoltzmann equations. Physics of Fluids, 29(4), Article 046103.

https://doi.org/10.1063/1.4981227

All material supplied via JYX is protected by copyright and other intellectual property rights, and duplication or sale of all or part of any of the repository collections is not permitted, except that material may be duplicated by you for your research use or educational purposes in electronic or print form. You must obtain permission for any other use. Electronic or print copies may not be offered, whether for sale or otherwise to anyone who is not an authorised user. 
This manuscript was accepted by Phys. Fluids. Click here to see the version of record.

High-order regularization in LBEs

\title{
High-order regularization in lattice-Boltzmann equations
}

\author{
Keijo K. Mattila, ${ }^{1,}$ a) Paulo C. Philippi, ${ }^{2}$ and Luiz A. Hegele Jr. ${ }^{3}$ \\ 1) Department of Physics, University of Jyväskylä, P.O. Box 35 (YFL), \\ FI-40014 University of Jyväskylä, Finland and Department of Physics, \\ Tampere University of Technology, P.O. Box 692, FI-33101 Tampere, \\ Finland \\ 2) Mechanical Engineering Graduate Program, Pontifical Catholic University of Paraná, \\ 80215-901 Curitiba, PR, Brazil \\ 3) Department of Petroleum Engineering, State University of Santa Catarina, \\ 88330-668 Balneário Camboriú, SC, Brazil
}

(Dated: 3 April 2017)

A lattice-Boltzmann equation (LBE) is the discrete counterpart of a continuous kinetic model. It can be derived using a Hermite polynomial expansion for the velocity distribution function. Since LBEs are characterized by discrete, finite representations of the microscopic velocity space, the expansion must be truncated and the appropriate order of truncation depends on the hydrodynamic problem under investigation. Here we consider a particular truncation where the non-equilibrium distribution is expanded on a par with the equilibrium distribution, except that the diffusive parts of high-order non-equilibrium moments are filtered, i.e. only the corresponding advective parts are retained after a given rank. The decomposition of moments into diffusive and advective parts is based directly on analytical relations between Hermite polynomial tensors. The resulting, refined regularization procedure leads to recurrence relations where high-order non-equilibrium moments are expressed in terms of low-order ones. The procedure is appealing in the sense that stability can be enhanced without local variation of transport parameters, like viscosity, or without tuning the simulation parameters based on embedded optimization steps. The improved stability properties are here demonstrated using the perturbed double periodic shear layer flow and the Sod shock tube problem as benchmark cases.

\section{INTRODUCTION}

When a fluid flows, molecular properties (like momentum and kinetic energy) are transferred by two kind of mechanisms: diffusion and advection. Diffusion is related to the random motion of molecules and is responsible for entropy growth. In the reverse sense, advection means organization: molecular properties are transferred with the bulk flow, i.e. along the streamlines. Diffusion is dominant in the small Reynolds number $(R e)$ regime, but when $R e$ increases, diffusion becomes less effective and properties are mainly transferred by advection. Moreover, from a statistical point of view, fast relaxation to an equilibrium state corresponds to a low kinematic viscosity. So, in low-viscous fluids the interactions between the particles are so numerous or effective that momentum and energy between neighboring particles is quickly balanced; the system remains close to the equilibrium state. As a result, the net-flux of momentum is small in the moving frame of reference or, in other words, transport of momentum is sustained mainly by advection rather than by diffusion, which conforms to a high Re flow. This aspect is used here as the guiding principle when designing particular lattice-Boltzmann (LB) schemes for the numerical simulation of fluid flows with improved stability properties.

The lattice-Boltzmann method was introduced by McNamara et al. ${ }^{1}$, first as an improvement to the lattice-gas automata. Soon after, Higuera et al. ${ }^{2}$ considered stability properties

\footnotetext{
a) keijo.mattila@jyu.fi
} 
of a novel collision operator involving a set of relaxation parameters. Since then improving stability of LB schemes has been addressed by several authors and various solutions have been proposed. For recent reviews, see e.g. Refs. 3 and 4, and the references therein. The first solutions were based on the use of multiple relaxation times (MRT) tuned with the help of a linear stability analysis ${ }^{5-9}$. The entropic LBGK scheme ${ }^{10}$ appears as a second solution and was conceived based on the maximization of entropy by locally tuning the single relaxation time of the BGK collision mode ${ }^{11}$. Recently, a new extension of LB schemes was proposed, namely the entropic stabilizer ${ }^{12}$. Unlike the entropic LBGK scheme, the entropic stabilizer does not locally alter the viscosity, but rather relies on modifying the relaxation time for the higher-order moments (i.e. the moments beyond the stress tensor) which do not contribute to the viscosity. In this respect, this extension is akin to the already mentioned relaxation parameter tuning for MRT schemes.

Stability of LB schemes can also be improved by adopting larger discrete velocity sets. This option was investigated by Siebert et al. ${ }^{13}$. Another alternative is to add high-order Hermite polynomial tensors to the equilibrium distribution, trying to reduce the effect of their related moments on stability. This approach is commonly used in MRT and entropic models and was utilized by Ref. 13 for improving stability of the usual D2Q9 latticeBoltzmann equation (LBE). One can also resort to regularized LBEs, i.e. rewrite a LBE in such a manner as to filter the undesirable ghost moments from the numerical scheme (see e.g. Ref. 14 for a further discussion on the ghost moments). This choice was investigated by Ladd ${ }^{15}$, Chen et al. ${ }^{16}$, Zhang et al. ${ }^{17}$, and by Latt and Chopard ${ }^{18}$ for the D2Q9 model; very recently Mattila et al. ${ }^{19}$ compared this regularization with the entropic stabilizer. Here we propose a method for dealing with general flow problems which require utilization of high-order LBEs: the method is based on a systematic improvement of the standard regularization.

Namely, both the equilibrium $f^{e q}$ and non-equilibrium $f^{n e q}$ distributions are expanded on a finite Hermitian basis in this method: the non-equilibrium distribution is expanded in terms of non-equilibrium convective (or raw) moments which can be decomposed, each separately, into advective and diffusive parts. The diffusive parts are defined as the peculiar or central moments of the non-equilibrium distribution and hence they are related to the flux of momentum, energy, and other high-order moments in the moving frame of reference. The advective parts are polynomials of local flow velocities with non-equilibrium moments of lower order as coefficients. They do not have any contribution to the flow when the local velocity is null, but become important in high Mach number $(M a)$ flows.

Then comes our pivotal proposal: the expansion of the non-equilibrium distribution is carried out on a par with the equilibrium distribution, but the diffusive parts are retained only up to the last physically relevant non-equilibrium moment. Beyond that, only the advective parts are maintained while the diffusive parts are filtered out which leads to specific recurrencerelations expressing the high-order non-equilibrium moments in terms of the local flow velocity and lower rank non-equilibrium moments. Technically the above procedure corresponds to regularization in the peculiar (or central) moment space. The procedure is conveniently and efficiently implemented using the recurrence relations here derived. Note that the usual regularization procedure operates with the convective moments and, therefore, filters both the diffusive and advective parts of the high-order non-equilibrium moments.

The proposed procedure is motivated by the essential role of advective transport when both $M a$ and $R e$ have large enough values. The procedure is general, directly applicable to high-order velocity sets, and relies purely on Hermite polynomial expansions of the distribution function. Thus, there is no need to tailor a moment space representation for the populations $f_{i}$ related to a particular discrete velocity set by, e.g., resorting to the Gram-Schmidt orthogonalization procedure. This advantage is already recognized (see e.g. Refs. 17, 20-23). Moreover, Dubois et al. ${ }^{24,25}$ recently showed that the choice of moments does influence the stability properties of a LB scheme: a poor choice of moments can lead to suboptimal stability ranges. Therefore tailoring moments in an ad-hoc manner, or even arbitrarily, seems problematic, especially in the case of large discrete velocity sets; we pursue 
a general solution for improving the stability of LB schemes.

We start by presenting the fundamentals of kinetic models and Hermite polynomial expansions of the velocity distribution in Sec. II. Particularly, we consider finite Hermitepolynomial expansion of distributions using central moments: the decomposition of moments into advective and diffusive parts is presented together with a relation expressing the raw (or convective) Hermite moments in terms of the diffusive parts. This relation is critical for the regularization procedure here proposed. Section III establishes the general principle for representing non-equilibrium moments in a subspace $\mathcal{H}_{q}$ - an important aspect when considering a general regularization procedure for high-order LBEs. Lattice-Boltzmann schemes are then considered in Sec. IV: the standard or original regularization procedure is first reviewed, from the high-order LBE perspective, after which the new, refined regularization procedure, where only the diffusive parts are filtered, is presented. Numerical experiments, using the perturbed double periodic shear layer flow and the Sod shock tube as benchmark cases, are reported in Sec. V. Conclusions are presented at the end.

\section{KINETIC PROJECTION}

We consider the Boltzmann equation or, more generally, a kinetic model equation for the distribution $f(\boldsymbol{x}, \boldsymbol{c}, t)$,

$$
\partial_{t} f+c \cdot \nabla_{x} f=\Omega
$$

which admits an equilibrium solution, namely the-Maxwell-Boltzmann (MB) distribution ${ }^{26-28}$,

$$
f^{\mathrm{MB}}=\frac{\rho}{\xi_{0}^{D}}[2 \pi(\Theta+1)]^{-\frac{D}{2}} \exp \left[-\frac{\|\boldsymbol{\xi}-\overline{\boldsymbol{u}}\|^{2}}{2(\Theta+1)}\right]
$$

where $\rho$ is the mass density of particles, $D$ is the Euclidean dimension of the velocity space, $\xi_{0}=\sqrt{k T_{0} / m}$ is a thermal reference speed, $k$ is the Boltzmann constant, $T_{0}$ is a reference temperature, $m$ is the mass of each particle, and $\|\cdot\|$ denotes the length (or Euclidean norm) of a vector. Furthermore, $\overline{\boldsymbol{u}}=\boldsymbol{u} / \xi_{0}$ and $\boldsymbol{\xi}=\boldsymbol{c} / \xi_{0}$ are dimensionless flow and molecular velocities, respectively, while $\Theta=T / T_{0}-1$ is the relative deviation from the reference temperature. The dimensionless mass density $\varrho=\rho / \rho_{0}$ is defined using the reference mass density $\rho_{0}$. Finally, the dimensionless distribution function is defined as $\bar{f}=f \xi_{0}^{D} / \rho_{0}$.

In its simplest form, the interaction term $\Omega$ in Eq. (1) is specified by the BGK singlerelaxation time collision model ${ }^{11}, \Omega=-\left(f-f^{e q}\right) / \tau$, where $\tau$ is a relaxation time. The kinetic equation, Eq. (1), can then be written as

$$
\partial_{t} f+c \cdot \nabla_{x} f=-\frac{1}{\tau} f^{n e q},
$$

where $f^{n e q}=f-f^{e q}$ represents the non-equilibrium part of the distribution function.

\section{A. Hydrodynamic variables}

The single-relaxation time BGK collision model, Eq. (2), describes dynamics of fluids obeying the ideal gas equation of state. Specifically, let $\xi_{T}=\sqrt{k T / m}$, which implies $\xi_{T}^{2}=\xi_{0}^{2}(\Theta+1)$. Furthermore, the mass, momentum, energy, and internal energy densities are defined as moments of the distribution function:

$$
\begin{aligned}
\rho & =\int f \mathrm{~d} \boldsymbol{c}, \quad \rho \boldsymbol{u}=\int \boldsymbol{c} f \mathrm{~d} \boldsymbol{c}, \\
\rho e & =\frac{1}{2} \int\|\boldsymbol{c}\|^{2} f \mathrm{~d} \boldsymbol{c}, \quad \rho e_{i n t}=\frac{1}{2} \int\|\boldsymbol{c}-\boldsymbol{u}\|^{2} f \mathrm{~d} \boldsymbol{c} .
\end{aligned}
$$




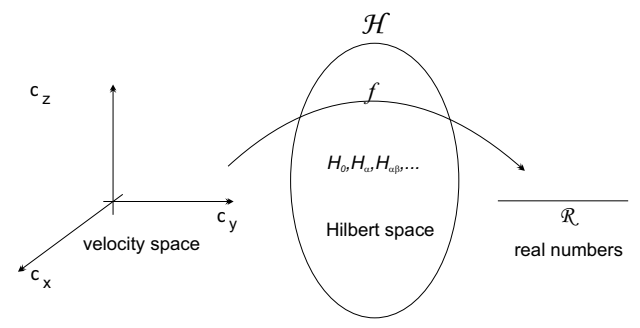

FIG. 1. The Hilbert space solution of the Boltzmann equation

Then, according to the equipartition theorem, the internal energy per unit mass is $e_{i n t}=$ $D \xi_{0}^{2}(\Theta+1) / 2$ which relates the relative deviation from the reference temperature to the internal energy. The ideal gas equation of state can be expressed in the form $p=\rho \xi_{0}^{2}(\Theta+1)$. The momentum flux tensor as well as the energy and heat flux vectors are also moments of the distribution function:

$$
\begin{aligned}
\Pi & =\int \boldsymbol{c} c f \mathrm{~d} \boldsymbol{c}, \quad \boldsymbol{E}=\frac{1}{2} \int \boldsymbol{c}\|\boldsymbol{c}\|^{2} f \mathrm{~d} \boldsymbol{c}, \\
\boldsymbol{q} & =\frac{1}{2} \int(\boldsymbol{c}-\boldsymbol{u})\|\boldsymbol{c}-\boldsymbol{u}\|^{2} f \mathrm{~d} \boldsymbol{c} .
\end{aligned}
$$

\section{B. Hermite polynomial expansion of the distribution}

For a given space-time point $(\boldsymbol{x}, t)$, the distribution $f(\boldsymbol{x}, \boldsymbol{c}, t)$ may be considered as a map from the continuous velocity space $\boldsymbol{c}$ onto the space $\mathcal{R}$ of real numbers (see Fig. 1). In fact, the distribution belongs to the Hilbert space $\mathcal{H}$ of square integrable functions $f: c \rightarrow \mathcal{R}$ and may be written in terms of an orthogonal basis of $\mathcal{H}$ that will be considered as the infinite set of Hermite polynomial tensors, i.e.

$$
\bar{f}(\boldsymbol{x}, \boldsymbol{c}, t)=\omega(\boldsymbol{\xi}) \sum_{j, k, l=0}^{\infty} \frac{a^{(j, k, l)}(\boldsymbol{x}, t) H^{(j, k, l)}(\boldsymbol{\xi})}{j ! k ! l !},
$$

where $\omega(\boldsymbol{\xi})=\exp \left(-\|\boldsymbol{\xi}\|^{2} / 2\right)(2 \pi)^{-D / 2}$. A component of a rank $r=j+k+l$ Hermite polynomial tensor $\boldsymbol{H}$ is denoted with $H^{(j, k, l)}$, where $j, k$, and $l$ indicate the number of times the coordinate direction $x, y$, and $z$ appears in the component index, respectively. For example, $H^{(0,0,0)} \equiv H_{0}, H^{(2,0,0)} \equiv H_{x x}$, and $H^{(1,1,1)} \equiv H_{x y z}$.

The first few Hermite polynomial tensors are

$$
\begin{aligned}
H_{0}(\boldsymbol{\xi})= & 1, \quad H_{\alpha}(\boldsymbol{\xi})=\xi_{\alpha}, \quad H_{\alpha \beta}(\boldsymbol{\xi})=\xi_{\alpha} \xi_{\beta}-\delta_{\alpha \beta} \\
H_{\alpha \beta \gamma}(\boldsymbol{\xi})= & \xi_{\alpha} \xi_{\beta} \xi_{\gamma}-\left(\xi_{\alpha} \delta_{\beta \gamma}+\xi_{\beta} \delta_{\alpha \gamma}+\xi_{\gamma} \delta_{\alpha \beta}\right) \\
H_{\alpha \beta \gamma \delta}(\boldsymbol{\xi})= & \xi_{\alpha} \xi_{\beta} \xi_{\gamma} \xi_{\delta}-\left(\xi_{\alpha} \xi_{\beta} \delta_{\gamma \delta}+\xi_{\alpha} \xi_{\gamma} \delta_{\beta \delta}+\xi_{\alpha} \xi_{\delta} \delta_{\beta \gamma}\right. \\
& \left.+\xi_{\beta} \xi_{\gamma} \delta_{\alpha \delta}+\xi_{\beta} \xi_{\delta} \delta_{\alpha \gamma}+\xi_{\gamma} \xi_{\delta} \delta_{\alpha \beta}\right)+\left(\delta_{\alpha \beta} \delta_{\gamma \delta}\right. \\
& \left.+\delta_{\alpha \gamma} \delta_{\beta \delta}+\delta_{\alpha \delta} \delta_{\beta \gamma}\right)
\end{aligned}
$$

when $\delta_{\alpha \beta}$ is the Kronecker delta. Furthermore, the following recurrence relation can be derived for the components of the Hermite polynomial tensors:

$$
H^{(j+1, k, l)}(\boldsymbol{\xi})=\xi_{x} H^{(j, k, l)}(\boldsymbol{\xi})-j \times H^{(j-1, k, l)}(\boldsymbol{\xi}), \quad j \geq 0,
$$

and similarly for $k$ and $l$. 


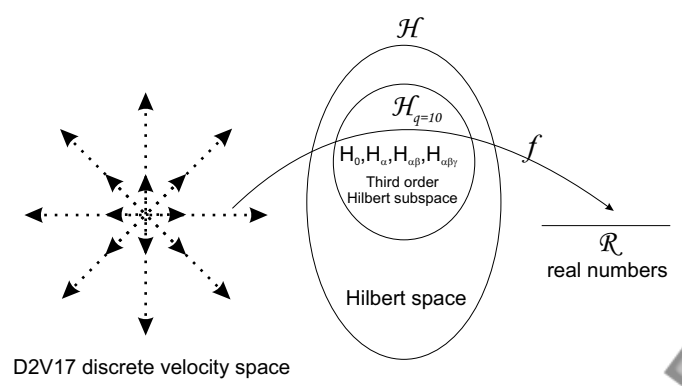

FIG. 2. Subspace $\mathcal{H}_{q=10}$ of $\mathcal{H}$ generated by a finite set of 10 Hermite polynomials and the conforming D2V17 discrete velocity set.

The Hermite polynomial tensors are orthogonal with respect to a weighted inner product:

$$
\int \omega(\boldsymbol{\xi}) H^{(j, k, l)}(\boldsymbol{\xi}) H^{(m, n, p)}(\boldsymbol{\xi}) \mathrm{d} \boldsymbol{\xi}=\delta_{j m} \delta_{k n} \delta_{l p} j ! k ! l ! .
$$

This orthogonality property, together with Eq. (3), provides a definition for the dimensionless expansion coefficient,

$$
a^{(m, n, p)}(\boldsymbol{x}, t)=\int \bar{f} H^{(m, n, p)}(\boldsymbol{\xi}) \mathrm{d} \boldsymbol{\xi}
$$

i.e. $a^{(m, n, p)}$ is a Hermite moment of the dimensionless distribution function.

\section{Projection onto a subspace: finite Hermite expansion}

Consider now the subspace $\mathcal{H}_{q}$ of $\mathcal{H}$ generated by a finite set of $q$ Hermite polynomials (see Fig. 2). The subspace $\mathcal{H}_{q}$ inherits the inner product from $\mathcal{H}$ which induces a metrics. Projection of the equilibrium solution, i.e. the Maxwell-Boltzmann distribution, onto this subspace results in

$$
\bar{f}^{e q}=\omega(\boldsymbol{\xi}) \sum_{H \in \mathcal{H}_{q}} \frac{a_{e q}^{(j, k, l)}(\boldsymbol{x}, t) H^{(j, k, l)}(\boldsymbol{\xi})}{j ! k ! l !} .
$$

In other words, $\bar{f}^{e q}$ is unambiguously determined by the equilibrium moments $a_{e q}^{(j, k, l)}$ given by Eq. (5) when evaluated with $\bar{f}^{\mathrm{MB}}$. The first few dimensionless equilibrium moments are

$$
\begin{aligned}
a_{0}^{e q}= & \varrho, \quad a_{\alpha}^{e q}=\varrho \bar{u}_{\alpha}, \quad a_{\alpha \beta}^{e q}=\varrho \Theta \delta_{\alpha \beta}+\varrho \bar{u}_{\alpha} \bar{u}_{\beta} \\
a_{\alpha \beta \gamma}^{e q}= & \varrho \Theta\left(\bar{u}_{\alpha} \delta_{\beta \gamma}+\bar{u}_{\beta} \delta_{\alpha \gamma}+\bar{u}_{\gamma} \delta_{\alpha \beta}\right)+\varrho \bar{u}_{\alpha} \bar{u}_{\beta} \bar{u}_{\gamma} \\
a_{\alpha \beta \gamma \delta}^{e q}= & \varrho \Theta^{2}\left(\delta_{\alpha \beta} \delta_{\gamma \delta}+\delta_{\alpha \gamma} \delta_{\beta \delta}+\delta_{\alpha \delta} \delta_{\beta \gamma}\right)+\varrho \Theta\left(\bar{u}_{\alpha} \bar{u}_{\beta} \delta_{\gamma \delta}\right. \\
& +\bar{u}_{\alpha} \bar{u}_{\gamma} \delta_{\beta \delta}+\bar{u}_{\alpha} \bar{u}_{\delta} \delta_{\beta \gamma}+\bar{u}_{\beta} \bar{u}_{\gamma} \delta_{\alpha \delta}+\bar{u}_{\beta} \bar{u}_{\delta} \delta_{\alpha \gamma} \\
& \left.+\bar{u}_{\gamma} \bar{u}_{\delta} \delta_{\alpha \beta}\right)+\varrho \bar{u}_{\alpha} \bar{u}_{\beta} \bar{u}_{\gamma} \bar{u}_{\delta} .
\end{aligned}
$$

The subspace $\mathcal{H}_{q}$ is directly related to the set of $n_{b}$ discrete velocities $\boldsymbol{c}_{i}, i=0, \ldots, n_{b}-1$, used to represent the microscopic velocity space. This formal relationship was found by Philippi and co-workers ${ }^{29,30}$ and is based on the requirement that a discrete Hermitian representation respects the orthogonality described by Eq. (4) up to a given order, i.e.

$$
\sum_{i=0}^{n_{b}-1} w_{i} H^{(j, k, l)}\left(\boldsymbol{\xi}_{i}\right) H^{(m, n, p)}\left(\boldsymbol{\xi}_{i}\right)=\delta_{j m} \delta_{k n} \delta_{l p} j ! k ! l !
$$


for all $H \in \mathcal{H}_{q}$, where $w_{i}$ are the discrete weight coefficients (see e.g. Refs. 21, 31, and 32 for further details). Therefore, LBEs with increasing order of approximation to a given kinetic model can be rigorously derived by adopting increasingly larger sets of Hermite polynomials leading to larger discrete velocity sets.

\section{Finite Hermite expansion with central moments}

In addition to targeting high-order LBEs, we also pursue stability improvements in the simulation of high $R e$ flows. Like discussed in the introduction, advection is the dominant transport mechanism in flows where both $M a$ and $R e$ have large values. This aspect suggests decomposition of a moment into advective and diffusive parts. The diffusive part is naturally defined as a central moment of the distribution, since it represents the moment in a moving frame of reference. Central moments are a standard concept in the kinetic theory and have been utilized, e.g., by Ruggeri ${ }^{33}$ and Torrilhonet al. ${ }^{34}$ in their treatment of extended thermodynamics.

First we note that the orthogonality of Hermite polynomials, Eq. (4), holds also with the relative or peculiar velocity $\boldsymbol{\xi}-\overline{\boldsymbol{u}}$, i.e.

$$
\begin{aligned}
& \int \omega(\boldsymbol{\xi}-\overline{\boldsymbol{u}}) H^{(j, k, l)}(\boldsymbol{\xi}-\overline{\boldsymbol{u}}) \\
& \quad \times H^{(m, n, p)}(\boldsymbol{\xi}-\overline{\boldsymbol{u}}) \mathrm{d} \boldsymbol{\xi}=\delta_{j m} \delta_{k n} \delta_{l p} j ! k ! l ! .
\end{aligned}
$$

An alternative expansion of the distribution relies on central moments,

$$
\bar{f}=\omega(\boldsymbol{\xi}-\bar{u}) \sum_{H \in \mathcal{H}_{q}} \frac{\widetilde{a}^{(j, k, l)}(\boldsymbol{x}, t) H^{(j, k, l)}(\boldsymbol{\xi}-\overline{\boldsymbol{u}})}{j ! k ! l !},
$$

where

$$
\widetilde{a}^{(m, n, p)}(\boldsymbol{x}, t):=\int \bar{f} H^{(m, n, p)}(\boldsymbol{\xi}-\overline{\boldsymbol{u}}) \mathrm{d} \boldsymbol{\xi} .
$$

That is, $\widetilde{a}^{(m, n, p)}$ represents the diffusive part of a moment and is defined as the peculiar (or central) Hermite moment of the distribution. The advective part is defined simply as

$$
\widehat{a}^{(m, n, p)}(\boldsymbol{x}, t):=a^{(m, n, p)}(\boldsymbol{x}, t)-\widetilde{a}^{(m, n, p)}(\boldsymbol{x}, t) .
$$

The diffusive parts of equilibrium moments are given by Eq. (11) evaluated with the Maxwell-Boltzmann distribution $\bar{f}^{\mathrm{MB}}$. The first few are

$$
\begin{aligned}
\widetilde{a}_{0}^{e q} & =\varrho, \quad \widetilde{a}_{\alpha \beta}^{e q}=\varrho \Theta \delta_{\alpha \beta}, \\
\widetilde{a}_{\alpha \beta \gamma \delta}^{e q} & =\varrho \Theta^{2}\left(\delta_{\alpha \beta} \delta_{\gamma \delta}+\delta_{\alpha \gamma} \delta_{\beta \delta}+\delta_{\alpha \delta} \delta_{\beta \gamma}\right) .
\end{aligned}
$$

Diffusive parts of equilibrium moments of odd rank are all identically zero. Furthermore, in isothermal flows, where $\Theta \equiv 0$, diffusive parts of all equilibrium moments vanish except $\tilde{a}_{0}^{e q}$. Finally, the advective parts of first equilibrium moments are

$$
\begin{aligned}
\widehat{a}_{\alpha}^{e q}= & \varrho \bar{u}_{\alpha}, \quad \widehat{a}_{\alpha \beta}^{e q}=\varrho \bar{u}_{\alpha} \bar{u}_{\beta}, \\
\widehat{a}_{\alpha \beta \gamma}^{e q}= & \varrho \bar{u}_{\alpha} \bar{u}_{\beta} \bar{u}_{\gamma}+\varrho \Theta\left(\bar{u}_{\alpha} \delta_{\beta \gamma}+\bar{u}_{\beta} \delta_{\alpha \gamma}+\bar{u}_{\gamma} \delta_{\alpha \beta}\right), \\
\widehat{a}_{\alpha \beta \gamma \delta}^{e q}= & \varrho \bar{u}_{\alpha} \bar{u}_{\beta} \bar{u}_{\gamma} \bar{u}_{\delta}+\varrho \Theta\left(\bar{u}_{\alpha} \bar{u}_{\beta} \delta_{\gamma \delta}+\bar{u}_{\alpha} \bar{u}_{\gamma} \delta_{\beta \delta}\right. \\
& \left.+\bar{u}_{\alpha} \bar{u}_{\delta} \delta_{\beta \gamma}+\bar{u}_{\beta} \bar{u}_{\gamma} \delta_{\alpha \delta}+\bar{u}_{\beta} \bar{u}_{\delta} \delta_{\alpha \gamma}+\bar{u}_{\gamma} \bar{u}_{\delta} \delta_{\alpha \beta}\right) .
\end{aligned}
$$


More generally, the relation

$$
\begin{aligned}
H^{(m, n, p)}(\boldsymbol{\xi})= & \sum_{j=0}^{m} \sum_{k=0}^{n} \sum_{l=0}^{p}\left(\begin{array}{c}
m \\
j
\end{array}\right)\left(\begin{array}{l}
n \\
k
\end{array}\right)\left(\begin{array}{l}
p \\
l
\end{array}\right) \\
& \times \bar{u}_{x}^{j} \bar{u}_{y}^{k} \bar{u}_{z}^{l} H^{(m-j, n-k, p-l)}(\boldsymbol{\xi}-\overline{\boldsymbol{u}}),
\end{aligned}
$$

written using binomial coefficients, allows to express the raw (or convective) Hermite moments in terms of the diffusive parts. Namely,

$$
a^{(m, n, p)}(\boldsymbol{x}, t):=\int \bar{f} H^{(m, n, p)}(\boldsymbol{\xi}) \mathrm{d} \boldsymbol{\xi},
$$

together with the relation in Eq. (15), expansion in Eq. (10), and the orthogonality property, Eq. (9), gives

$$
\begin{aligned}
a^{(m, n, p)}(\boldsymbol{x}, t)= & \left.\sum_{j=0}^{m} \sum_{k=0}^{n} \sum_{l=0}^{p}\left(\begin{array}{l}
m \\
j
\end{array}\right)\left(\begin{array}{l}
n \\
k
\end{array}\right)\left(\begin{array}{l}
p \\
l
\end{array}\right)\right) \\
& \times \bar{u}_{x}^{j} \bar{u}_{y}^{k} \bar{u}_{z}^{l} \widetilde{a}^{(m-j, n-k, p-l)}(\boldsymbol{x}, t) .
\end{aligned}
$$

The resulting expressions for the first few moments are

$$
\begin{aligned}
a_{0}= & \varrho, \quad a_{\alpha}=\varrho \bar{u}_{\alpha}, \quad a_{\alpha \beta}=\widetilde{a}_{\alpha \beta}+\varrho \bar{u}_{\alpha} \bar{u}_{\beta}, \\
a_{\alpha \beta \gamma}= & \widetilde{a}_{\alpha \beta \gamma}+\bar{u}_{\alpha} \widetilde{a}_{\beta \gamma}+\bar{u}_{\beta} \widetilde{a}_{\alpha \gamma}+\bar{u}_{\gamma} \widetilde{a}_{\alpha \beta}+\varrho \bar{u}_{\alpha} \bar{u}_{\beta} \bar{u}_{\gamma}, \\
a_{\alpha \beta \gamma \delta}= & \widetilde{a}_{\alpha \beta \gamma \delta}+\bar{u}_{\alpha} \widetilde{a}_{\beta \gamma \delta}+\bar{u}_{\beta} \widetilde{a}_{\alpha \gamma \delta}+\bar{u}_{\gamma} \widetilde{a}_{\alpha \beta \delta}+\bar{u}_{\delta} \widetilde{a}_{\alpha \beta \gamma} \\
& +\bar{u}_{\alpha} \bar{u}_{\beta} \widetilde{a}_{\gamma \delta}+\bar{u}_{\alpha} \bar{u}_{\gamma} \widetilde{a}_{\beta \delta}+\bar{u}_{\alpha} \bar{u}_{\delta} \widetilde{a}_{\beta \gamma}+\bar{u}_{\beta} \bar{u}_{\gamma} \widetilde{a}_{\alpha \delta} \\
& +\bar{u}_{\beta} \bar{u}_{\delta} \widetilde{a}_{\alpha \gamma}+\bar{u}_{\gamma} \bar{u}_{\delta} \widetilde{a}_{\alpha \beta}+\varrho \bar{u}_{\alpha} \bar{u}_{\beta} \bar{u}_{\gamma} \bar{u}_{\delta},
\end{aligned}
$$

where we have already utilized $\widetilde{a}_{0}=\varrho$ and $\widetilde{a}_{\alpha} \equiv 0$.

It is immediately confirmed that, in the case of equilibrium moments, these expressions agree with Eqs. (13) and (14). It is also worth noting that, in general, the advective parts depend on strictly lower-rank moments multiplied by powers of the local flow velocity (i.e. the advective parts vanish with $\boldsymbol{u}$ ). The relation in Eq. (16), and the expressions in (17), will be utilized below when proposing a regularization procedure where the diffusive parts of the high-order non-equilibrium moments are filtered. In the next section, however, we first establish the general principle for representing non-equilibrium moments in a subspace $\mathcal{H}_{q}$.

\section{IMPROVING THE HERMITIAN REPRESENTATION}

According to the usual multiple-scale Chapman-Enskog analysis, the leading-order of the non-equilibrium distribution in the BGK model, Eq. (2), is given by

$$
f^{(1)}=-\tau\left(\partial_{t_{0}} f^{e q}+\partial_{\alpha} c_{\alpha} f^{e q}\right)
$$

where we have assumed $f^{(0)}=f^{e q}$. The leading order of the viscous stress tensor $\tau_{\alpha \beta}$ is obtained by computing the second-order (central) moment of $f^{(1)}$ :

$$
\tau_{\alpha \beta}=\int f^{(1)}\left(c_{\alpha}-u_{\alpha}\right)\left(c_{\beta}-u_{\beta}\right) \mathrm{d} \boldsymbol{c}=\int f^{(1)} c_{\alpha} c_{\beta} \mathrm{d} \boldsymbol{c}
$$

after enforcing the usual solvability conditions.

From these two expressions it becomes apparent that in order to properly capture the leading order of the viscous stress tensor, the Hermitian basis of a subspace $\mathcal{H}_{q}$ should 


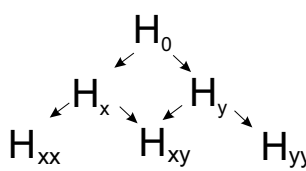

(a)

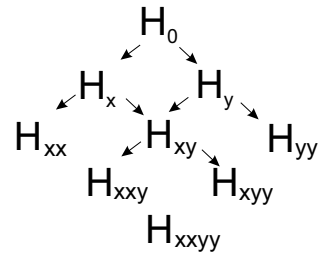

(b)

FIG. 3. Hermitian basis of the Hilbert subspaces (a) $\mathcal{H}_{q=6}$ and (b) $\mathcal{H}_{q=9}$ related to the D2Q9 LBE. Arrows from the components of rank $r$ Hermite polynomial tensors point to the components of rank $r+1$ Hermite polynomials tensors that are necessary to be in the Hermitian basis for the correct retrieval of the corresponding non-equilibrium moment. For example, in representation (b) the $x y$-component of the viscous stress tensor is the only component retrieved without errors (up to first-order in the usual multiple-scale Chapman-Enskog analysis).

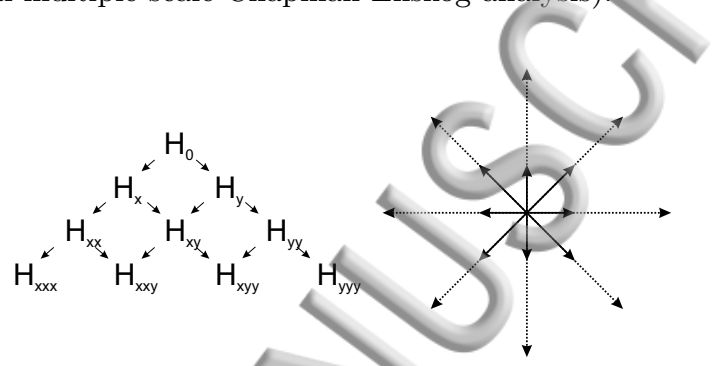

(a)

(b)

FIG. 4. Third-order Hermitian basis of the subspace $\mathcal{H}_{q=10}$ and the conforming D2V17 discrete velocity set.

include full Hermite polynomial tensors up to rank 3 (because $\tau_{\alpha \beta}$ depends on $\partial_{\gamma} a_{\alpha \beta \gamma}^{e q}$ ). This is clearly not the case for second-order representations. For concreteness, let us consider the usual Hermitian second-order basis of the D2Q9 LBE, composed of the 6 Hermite polynomials $H_{0}, H_{x}, H_{y}, H_{x x}, H_{x y}$, and $H_{y y}$ (see Fig. 3 ). In fact, it turns out that the D2Q9 velocity set, together with its associated weights $w_{i}$ and the scaling factor $a_{s}$, fulfills Eq. (8) even when the Hermite polynomials $H_{x x y}, H_{x y y}$, and $H_{x x y y}$ are included into the basis. Thus, the subspace $\mathcal{H}_{q=9}$ can be adopted equally well (see Fig. 3) ${ }^{13,29}$. This Hermitian basis for $\mathcal{H}_{q=9}$ can be obtained directly via Cartesian product between the two one-dimensional sets $H_{0}, H_{x}, H_{x x}$, and $H_{0}, H_{y}, H_{y y}$ (see e.g. Ref. 35).

Like depicted in Fig. 3, with the $\mathcal{H}_{q=9}$ subspace $\tau_{x y}$ is the only second-order nonequilibrium moment that is retrieved without errors (up to first-order in the usual multiplescale Chapman-Enskog analysis) since $H_{x x y}$ and $H_{x y y}$ indeed belong to the Hermitian basis of $\mathcal{H}_{q=9}$. At the same time, the moments $\tau_{x x}$ and $\tau_{y y}$ depend also on the equilibrium moments $a_{x x x}^{e q}$ and $a_{y y y}^{e q}$, i.e. the basis should also include $H_{x x x}$ and $H_{y y y}$ (which is not possible with the D2Q9 velocity set). In the case of $\mathcal{H}_{q=6}$, obviously none of the viscous stress tensor components is retrieved without errors. Both with $\mathcal{H}_{q=6}$ and $\mathcal{H}_{q=9}$, the above is manifested as $\mathcal{O}\left(u^{3}\right)$ errors in the macroscopic momentum balance equations - a recent investigation on the topic was presented by Dellar ${ }^{36}$. These errors can be avoided by using at least third-order representations like D2V17 (see Figs. 2 and 4).

Similarly, when modeling compressible and thermal flows, in order to correctly capture the third-order non-equilibrium moments with LBE, in particular the heat flux vector $q_{\alpha}$, a full fourth-order Hermitian representation for the basis of a subspace $\mathcal{H}_{q}$ is required. Generally speaking, rank $r$ non-equilibrium moments depend on the equilibrium moments up to rank $r+1$ and hence, in order to properly represent them in a subspace $\mathcal{H}_{q}$, the Hermitian basis of the subspace must include full Hermite polynomial tensors up to rank $r+1$. Or conversely, if a Hermitian basis includes full tensors up to rank $r$, the subspace $\mathcal{H}_{q}$ can maintain full non-equilibrium moments up to rank $r-1$ : in this case we say that 
rank $r$ non-equilibrium moments are not supported by the $\mathcal{H}_{q}$ subspace or, alternatively, that they do not fit into the Hermitian representation.

Note that the advective part of a rank $r$ non-equilibrium moment in fact fits into the Hermitian representation when the basis includes full tensors up to rank $r$ (see the end of Sec. IID). This is not the case with the corresponding diffusive part, however, because it depends on the rank $r+1$ equilibrium moments. To comply with the situation, an option is to expand the diffusive part only up to rank $r-1$ (or to the last physically relevant non-equilibrium moment) while the advective part is still fully expanded on a par with the equilibrium distribution. As a matter of fact, this is the essence of the refined regularization procedure presented below. Here is also the point of departure between the current and previously published, related approaches where also the diffusive part is expanded all the way up to rank $r$ (see e.g. Refs. 17, 20, and 37).

\section{LATTICE-BOLTZMANN SCHEMES}

Let us consider a discrete version of Eq. (2), namely the standard lattice-BGK (LBGK) equation:

$$
f_{i}\left(\boldsymbol{x}+h \boldsymbol{e}_{i}, t+\delta t\right)=f_{i}^{e q}(\boldsymbol{x}, t)+\left(1-\frac{\delta t}{\tau}\right) f_{i}^{n e q}(\boldsymbol{x}, t)
$$

where $f_{i}^{n e q}=f_{i}-f_{i}^{e q}, h$ is the lattice spacing, $\delta t$ the discrete time step, $\boldsymbol{e}_{i}=\boldsymbol{\xi}_{i} / a_{s}$ denote the lattice vectors, $a_{s}$ is the dimensionless scaling factor associated with a given discrete velocity set, and $i=0, \ldots, n_{b}-1$. For example, $a_{s}=\sqrt{3}$ in the D2Q9, D3Q19, and D3Q27 discrete velocity sets. Note that the discrete distribution functions are here considered dimensionless by default in ordêr to simplify the notation (i.e. the overline notation is dropped for discrete distributions)

Accordingly, the discrete equilibrium function is defined as

$$
f_{i}^{e q}=w_{i} \sum_{H \in \mathcal{H}_{q}} \frac{a_{e q}^{(j, k, l)} H^{(j, k, l)}\left(\boldsymbol{\xi}_{i}\right)}{j ! k ! l !},
$$

where the dimensionless equilibrium moments correspond with Eq. (7) and

$$
\begin{aligned}
\varrho & :=\sum_{i=0}^{n_{b}-1} f_{i}, \quad \varrho \overline{\boldsymbol{u}}:=\sum_{i=0}^{n_{b}-1} f_{i} \boldsymbol{\xi}_{i} \\
\varrho \bar{e}_{\text {int }} & :=\frac{1}{2} \sum_{i=0}^{n_{b}-1} f_{i}\left\|\boldsymbol{\xi}_{i}-\overline{\boldsymbol{u}}\right\|^{2}, \quad \Theta=\frac{2}{D} \bar{e}_{\text {int }}-1 .
\end{aligned}
$$

Note that in isothermal flows $\Theta \equiv 0$ is used, and that here all discrete moments are computed using $\boldsymbol{\xi}_{i}$, i.e. not using $\boldsymbol{e}_{i}$. Furthermore, the scaling factor is related to the thermal reference speed, i.e. $\xi_{0}=c_{r} / a_{s}$, where $c_{r}=h / \Delta t$ is the lattice reference velocity (which originates from the relation $\boldsymbol{e}_{i}=\boldsymbol{c}_{i} / c_{r}$ ). The speed of sound with the LBGK scheme is $c_{s}=\sqrt{\gamma_{c} \xi_{0}(\Theta+1)}$ and $c_{s}^{i s o}=\sqrt{\xi_{0}}$ for thermal and isothermal flows, respectively: the heat capacity ratio is $\gamma_{c}=(D+2) / D$ in the case of monoatomic fluids with an ideal gas equation of state.

\section{A. Regularization}

Broadly speaking, regularization is a procedure applied with many numerical CFD methods in order to soothe the effect of errors that are produced in the course of a simulation. In the LB context, regularization is considered as a method for filtering the high-order 
non-equilibrium moments that do not fit into the Hermitian representation (or are beyond the macroscopic balance equations targeted). More specifically, in the standard LBGK scheme the equilibrium part is projected onto the subspace $\mathcal{H}_{q}$, but the non-equilibrium part is not: the distribution $f_{i}$ evolves according to Eq. (18) which allows the high-order non-equilibrium moments to persist in an unspecified manner. The regularization procedure addresses this issue by projecting also the relevant, low-order non-equilibrium moments onto $\mathcal{H}_{q}$ while filtering the high-order non-equilibrium moments which are beyond the targeted hydrodynamics.

This is accomplished by replacing $f_{i}^{n e q}$ in Eq. (18) with a finite Hermite expansion of the non-equilibrium distribution,

$$
f_{i}^{n e q}:=w_{i} \sum_{H \in \mathcal{H}_{q}} \frac{a_{n e q}^{(j, k, l)} H^{(j, k, l)}\left(\boldsymbol{\xi}_{i}\right)}{j ! k ! l !}
$$

where $a_{\text {neq }}^{(j, k, l)}=0$ is enforced for $j+k+l>R$ with a given rank $R$. In the case of isothermal flows, $R=2$ and Eq. (19) reduces to

$$
f_{i}^{n e q}:=\frac{w_{i}}{2} \bar{\tau}_{\alpha \beta} H_{\alpha \beta}\left(\boldsymbol{\xi}_{i}\right),
$$

with $a_{\alpha \beta}^{n e q}=\bar{\tau}_{\alpha \beta}$, and where Einstein summation convention is implied by the repeated indexes. The dimensionless non-equilibrium moments, which fulfill $m+n+p \leq R$, are computed at the pre-collision state (i.e. immediately after the propagation step) using

$$
a_{n e q}^{(m, n, p)}:=\sum_{i}\left[f_{i}-f_{i}^{e q}\right] H^{(m, n, p)}\left(\boldsymbol{\xi}_{i}\right) .
$$

This regularization was investigated by Latt and Chopard ${ }^{18}$ for the second-order D2Q9 LBE: they observed that the regularization improved the stability range of the LBGK scheme by a factor of 7.7 in the case of a lid-driven cavity flow. Montessori et al. ${ }^{38}$ applied the second-order regularized LB scheme for the three-dimensional lid-driven cavity flow (isothermal case) and observed that an order of magnitude reduction in CPU time could be achieved, compared to the standard LBGK scheme, due to the improved stability range.

Mattila and co-workers ${ }^{19}$ investigated the regularization, again for the D2Q9 LBE, by setting up a perturbed double periodic shear layer flow as a benchmark case. In this kind of problem, a small velocity perturbation, perpendicular to the shear flow direction, initiates a Kelvin-Helmholtz instability and causes roll-up of the anti-parallel shear layers that is dissipated along the time. They compared the regularized scheme with the standard LBGK scheme and the LBGK entropic stabilizer scheme ${ }^{12}$ (see Ref. 19 for details of the comparison).

Furthemore, Zhang et al. ${ }^{17}$, Niu et al. ${ }^{39}$, and Montessori et al. ${ }^{23}$ investigated simulation of finite $K n$ channel flows, i.e. Couette and Poiseuille flows, both in 2D and 3D, using third-order LBEs together with the regularization: they projected also the rank 3 nonequilibrium moments onto the subspaces $\mathcal{H}_{q}$ which, however, does not comply with the general principle established in Sec. III for representing non-equilibrium moments in terms of a finite Hermitian basis.

\section{B. Refined regularization: Filtering central moments}

The regularization procedure presented above filters the raw or convective high-order non-equilibrium moments that are beyond the macroscopic balance equations targeted (or do not fit into the Hermitian representation). For isothermal and thermal models this means that all raw or convective non-equilibrium moments higher than rank 2 and 3, respectively, are filtered. A more refined approach is also possible, where only selective parts of the highorder non-equilibrium moments are filtered. Such a procedure will be presented below. 
Equations (13), (14), and (17) clearly illustrate how the diffusive part is a representation of a moment in the moving frame of reference, while the advective part accounts for the bulk flow contribution to a moment (i.e. the part remaining after the diffusive part is subtracted from a convective moment). We therefore have an opportunity to acknowledge the essential role of advective transport in high $M a$ and Re flows. Namely, instead of filtering the convective high-order non-equilibrium moments, like in the original regularization procedure, we propose to filter only the diffusive parts; the aim is to retain the advective parts to the fullest extent possible with the adopted decomposition.

To begin with, the discrete counterpart of Eq. (11) is

$$
\widetilde{a}_{n e q}^{(m, n, p)}:=\sum_{i}\left[f_{i}-f_{i}^{e q}\right] H^{(m, n, p)}\left(\boldsymbol{\xi}_{i}-\overline{\boldsymbol{u}}\right),
$$

which is to be evaluated at the pre-collision state. Then the refined regularization procedure for filtering only the diffusive parts is very simple:

1. expand the non-equilibrium distribution still using Eq. (19), but now

2. the (convective) non-equilibrium moments are given by the relation Eq. (16), or Eq. (17), where

3. all the diffusive parts are omitted, and nothing else, after a given rank $R$; the remaining diffusive parts are computed using Eq. (20).

Note the crucial differences to the original regularization procedure. For example, in the case of isothermal flows, where all diffusive parts are filtered after rank 2, Eq. (17) gives

$$
\begin{aligned}
a_{\alpha \beta \gamma}^{n e q}= & \bar{u}_{\alpha} \bar{\tau}_{\beta \gamma}+\bar{u}_{\beta} \bar{\tau}_{\alpha \gamma}+\bar{u}_{\gamma} \bar{\tau}_{\alpha \beta}, \\
a_{\alpha \beta \gamma \delta}^{n e q}= & \bar{u}_{\alpha} \bar{u}_{\beta} \bar{\tau}_{\gamma \delta}+\bar{u}_{\alpha} \bar{u}_{\gamma} \bar{\tau}_{\beta \delta}+\bar{u}_{\alpha} \bar{u}_{\delta} \bar{\tau}_{\beta \gamma} \\
& +\bar{u}_{\beta} \bar{u}_{\gamma} \bar{\tau}_{\alpha \delta}+\bar{u}_{\beta} \bar{u}_{\delta} \bar{\tau}_{\alpha \gamma}+\bar{u}_{\gamma} \bar{u}_{\delta} \bar{\tau}_{\alpha \beta},
\end{aligned}
$$

where we have used $a_{\alpha \beta}^{n e q} \equiv \widetilde{a}_{\alpha \beta}^{n e q}=\bar{\tau}_{\alpha \beta}$. The resulting non-equilibrium moments are negligible in creeping flows but, on the other hand, can be significant in high Re flows. Finally, in thermal flows the heat flux vector is related to the diffusive parts of rank 3 nonequilibrium moments, i.e. $\bar{q}_{\alpha}=\frac{1}{2} \widetilde{a}_{\alpha \beta \beta}^{\text {neq }}$, where the repeated indexes again imply Einstein summation convention.

The expressions in Eq. (21) agree with the expressions derived by Malaspinas ${ }^{22}$ from a different premise. Moreover, Malaspinas restricted his analysis to isothermal flows and derived the expressions for the specific, second-order LBEs using D2Q9 and D3Q27 velocity sets. Interestingly, Malaspinas carried out a linear stability analysis for the resulting schemes: the analysis revealed that the new schemes indeed exhibit improved stability ranges. This appears to verify that the rationale behind filtering only the diffusive parts, and thus retaining the advective parts, is both reasonable and useful as a guiding principle when constructing stable LB schemes.

On the other hand, the refined regularization procedure presented here, and the related expressions, rely purely on Hermite polynomial expansions of the distribution function. The consequence of this systematic approach is that moment space representations of the distributions are immediately available (defined by the coefficients of the expansion). Recent studies highlight the importance of this aspect: Dubois et al. ${ }^{24,25}$ showed that the choice of moments does influence the stability properties of a LB scheme (e.g. a poor choice of moments can lead to suboptimal stability ranges). Therefore the alternative approach of tailoring moment space representations in an ad-hoc manner, or even arbitrarily, seems problematic when considering a general solution for improving the stability of LB schemes. To summarize, the above presented regularization procedure can be used both in $2 \mathrm{D}$ and $3 \mathrm{D}$, it is not restricted to a specific discrete velocity set, and, in particular, it can be used both with high-order LBEs and thermal flows. 


\section{NUMERICAL EXPERIMENTS}

To demonstrate the improved stability properties of the refined regularization procedure, we present simulation results for two benchmark cases: the perturbed double periodic shear layer flow and the Sod shock tube flow are simulated using the second-order D2Q9 and fourth-order D2V37 discrete velocity sets, respectively. These benchmark cases are chosen because they allow investigation of stability properties without the influence of particular fluid-solid or inlet/outlet boundary conditions, i.e. the two cases provide ideal settings for observing the effect of the collision operator alone. Moreover, the two cases are appropriate benchmark cases for stability as they involve significant, sudden variations in density and velocity fields (both in time and space).

\section{A. Perturbed double periodic shear layer flow}

In this two-dimensional flow configuration, periodic in both directions, two antiparallel shear layers are set up. Then, a small velocity perturbation is introduced, perpendicular to the shear flow directions, which causes roll up of the antiparallel shear layers and leads to spiral-like vorticity patterns. The initial velocity field, which includes the shear layers and the perturbation, is given by

$$
\begin{array}{ll}
u_{x}= \begin{cases}U_{0} \tanh \left[\lambda\left(\frac{y}{L}-\frac{1}{4}\right)\right], & y \leq \frac{L}{2}, \\
U_{0} \tanh \left[\lambda\left(\frac{3}{4}-\frac{y}{L}\right)\right], & y>\frac{L}{2},\end{cases} \\
u_{y} \neq \epsilon U_{0} \sin \left[2 \pi\left(\frac{x}{L}+\frac{1}{4}\right)\right] ;
\end{array}
$$

$U_{0}$ defines the amplitude of the initial velocities. In our simulations, $x$ and $y$ refer to node index coordinates while $L$ is the number of nodes in both $\mathrm{x}$ - and y-direction. Parameters $\lambda$ and $\epsilon$ control the width of the shear layer and the initial velocity perturbation, respectively. The Reynolds number is defined as $R e=U_{0} L / \nu$ and the characteristic time $T=L / U_{0}$.

The initial pressure field, conforming with the above initial velocity field, is solved from the pressure-Poisson equation (for an incompressible fluid). Furthermore, the nonequilibrium distributions are initialized as described in Ref. 19, i.e. the initial stress tensor is first determined from the strain rate tensor and then projected onto the kinetic space - the gradients of the velocity field are estimated using isotropic, second-order accurate finite-differences. Finally, when utilizing the refined regularization procedure, the recurrence relations are enforced already at the initialization of the distributions. Figure 5 presents snapshots of the vorticity field simulated using the recurrence relations and the D2Q9 discrete velocity set: in this simulation $\lambda=80$ and $\epsilon=0.05$, called a thin layer case according to Refs. 40 and 41, while $L=1024, U_{0}=64 / 1024$, and $R e=150000$.

We set out to compare stability properties of three LB relaxation procedures, namely the BGK, standard regularization, and refined regularization with recurrence relations, by searching for the maximum attainable Reynolds number for a given resolution and initial velocity $U_{0}$ (or Mach number defined as $M a=U_{0} / \xi_{0}$ ). Each relaxation procedure is used together with the D2Q9 discrete velocity set and the full $\mathcal{H}_{q=9}$ Hermite moment space representation (see Appendix for detailed descriptions of the LB schemes). Moreover, the maximum Reynolds numbers are searched for a thick layer case specified by $\lambda=30$ and $\epsilon=0.05$. A simulation is considered stable if it reaches $t=10 T$ without breaching the stability criteria.

Instead of simply waiting for a catastrophic divergence of a simulated solution, we adopt a more stringent interpretation of stability and impose two stability criteria: 1 . the kinetic energy in the system must decrease every discrete time step and 2. the pressure (or density) 

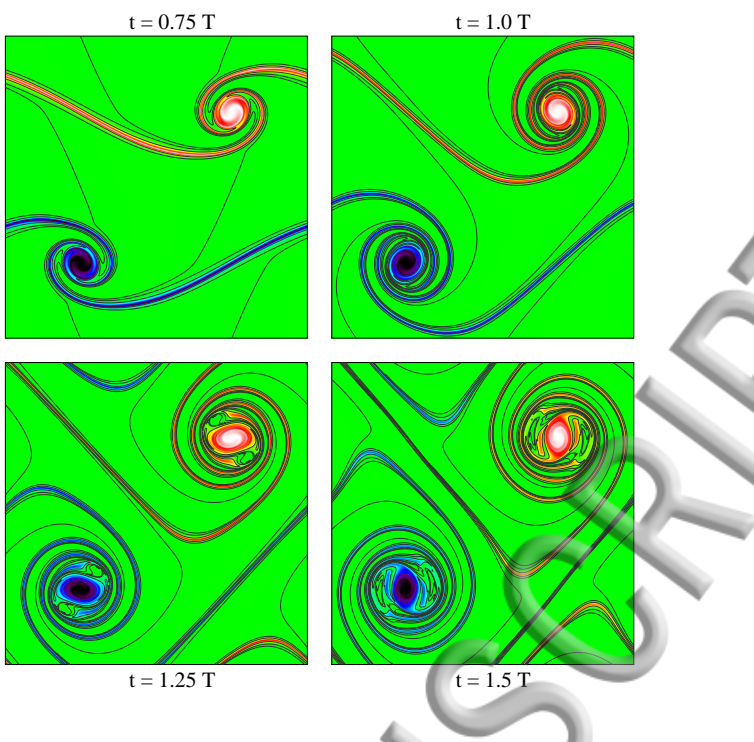

FIG. 5. Snapshots of the vorticity field in a perturbed double periodic shear layer flow simulation ( $\lambda=80$ and $\epsilon=0.05$, i.e. a so-called thin layer case according to Refs. 40 and 41). The simulation was executed using the recurrence relations with the D2Q9 discrete velocity set: $L=1024, U_{0}=$ $64 / 1024$, and $R e=150000$.

field must remain relatively smooth without artificial fluctuations or oscillations. The first criteria is not imposed during the initial stages of the simulation, specifically when $t \leq T / 10$, because of the inevitable disturbances caused by the start-up. The second criteria is enforced by counting the number of local extrema in the simulated density field (i.e. the sites where the density is either strictly greater or smaller than in all the neighboring lattice sites). After a visual inspection of numerous density fields simulated with various resolutions and $R e$, we determined that the local density extrema count must be less or equal to 50 at all times.

The final hurdle in constructing stability maps for the three relaxation procedures is related to their behavior at the incompressible limit (the Mach number decreases for a fixed $L$ and $R e$ ). Dellar ${ }^{7}$ considered convergence of the simulated solutions at the incompressible limit and observed that solutions computed with the BGK relaxation term indeed converge. However, with particular MRT schemes, essentially corresponding to the regularization procedure, the solutions diverge after $M a$ attains small enough values (the specific value depends on the resolution and $R e$ ). We repeated this experiment and the results are presented in Fig. 6: initially, starting form high $M a$, the solution converges for all schemes at the rate $M a^{2}$, but when reaching smaller $M a$, the divergence of solutions computed with the standard and refined regularization procedures is evident.

Without going into the reasons leading to the divergence, we simply acknowledge the fact and consider the point of divergence, dependent on both $L$ and $R e$, as an operational lower limit for $U_{0}$ when utilizing a regularization procedure. Also an upper limit for $U_{0}$ can be considered. Namely, at large enough $M a$ the compressibility effects start to influence and eventually dominate the solutions (see e.g. Fig. 1 in Ref. 42). A limiting value corresponding to $U_{0} \approx 0.1$ is commonly presented in the literature (see e.g. Ref. 43 and references therein). Interestingly, when approaching large values for $U_{0}$ in our search for maximum attainable $R e$, we observe a point after which the results coincide for the BGK relaxation term and the refined regularization with recurrence relations (see Figs. 7 and 8). We attribute this behavior to the compressibility effects and consider this particular point as an operational upper limit for $U_{0}$.

The shaded regions in Figs. 7 and 8 indicate the operational domains of regularization procedures (specified by the above discussed lower and upper limits for $U_{0}$ ). Furthermore, 


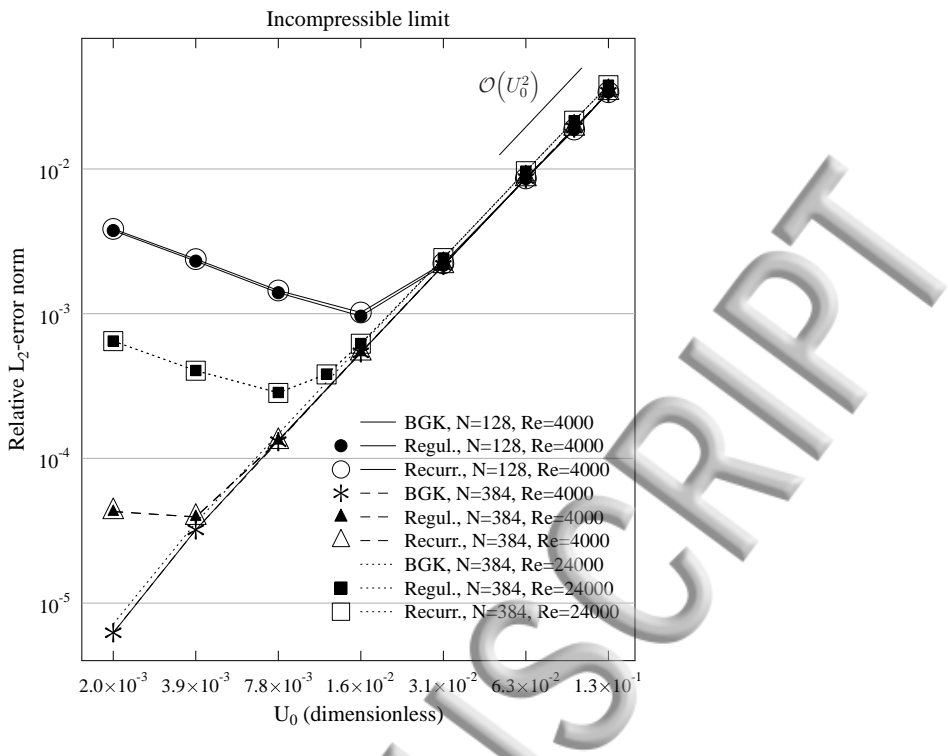

FIG. 6. Convergence of the relative L2-error norm in the incompressible limit, i.e. the Mach number is decreased for a fixed $L$ and $R e$. The error is computed for the local speed normalized with $U_{0}$, and the results are shown for three relaxation procedures utilized together with the D2Q9 velocity set and the full $\mathcal{H}_{q=9}$ Hermite representation. Reference solutions for $L=128$ and $R e=4000$, $L=384$ and $R e=4000$, as well as for $L=384$ and $R e=24000$, are all computed using the BGK relaxation term and $U_{0}=1 / 1024 \approx 9.77 \times 10^{-4}$.

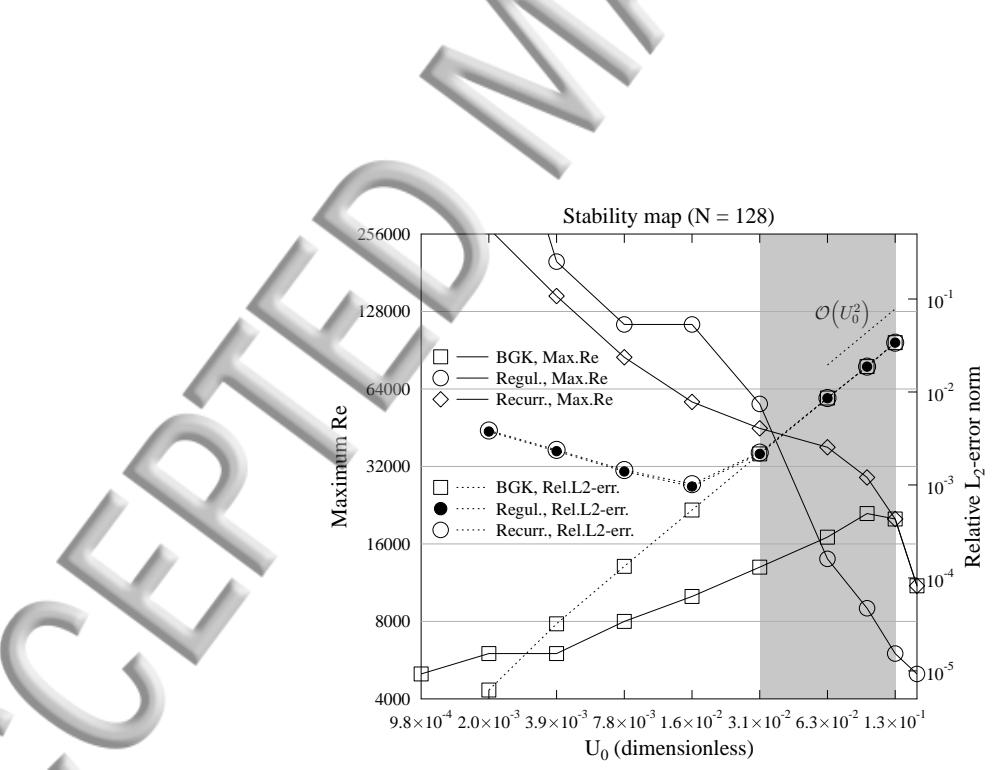

FIG. 7. The maximum attainable Reynolds numbers for three relaxation procedures utilized together with the D2Q9 velocity set and the full $\mathcal{H}_{q=9}$ Hermite representation. The maximum $R e$ is reported (left axis) as a function of $U_{0}$ and $L=128$. Also plotted is the relative L2-error norm (right axis; incompressible limit with $L=128$ and $R e=4000$, further details are explained in the caption of Fig. 6). The shaded region indicates the operational domain of regularization procedures (specified in the text). The recurrence relations provide a clear stability improvement across this $U_{0}$ range. 


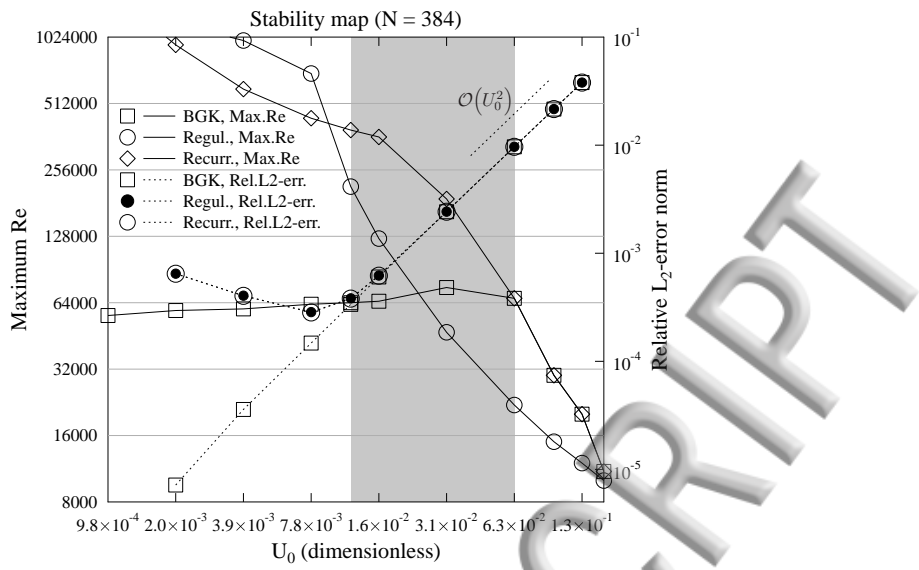

FIG. 8. The maximum attainable Reynolds numbers for three relaxation procedures utilized together with the D2Q9 velocity set and the full $\mathcal{H}_{q=9}$ Hermite representation. The maximum $R e$ is reported (left axis) as a function of $U_{0}$ and $L=384$. Also plotted is the relative L2-error norm (right axis; incompressible limit with $L=384$ and $R e=24000$, further details are explained in the caption of Fig. 6). The shaded region indicates the operational domain of regularization procedures (specified in the text). The recurrence relations provide a clear stability improvement across this $U_{0}$ range.

as presented in Figs. 7 and 8, the recurrence relations provide a clear stability improvement across the operational $U_{0}$ range. The stability maps also confirm that the advective parts of high-order non-equilibrium moments are essential when $M a$ and $R e$ attain large enough values: the standard regularization procedure filters these advective parts and, at the same time, presents low stability at high $M a$ values, i.e. towards the right border of the shaded area in Figs. 7 and 8. On the other hand, the diffusive parts of high-order non-equilibrium moments indeed appear to be responsible for stability problems: only the BGK relaxation term does not filter the diffusive parts and presents low stability at low $M a$ values (when diffusive parts dominate over advective parts).

The fact that the operational upper limit for $U_{0}$ has a smaller value with a better resolution is consistent with the compressibility effects ${ }^{42}$. Finally, the operational $U_{0}$ range for regularization procedures appears to widen when resolution is improved. Our implementations of the three relaxation procedures are computationally equally efficient, practically speaking, deviating in efficiency no more than $10-15 \%$.

\section{B. Sod shock tube flow}

In this setup, a compressible fluid with a discontinuity in density is considered. Initially the fluid is at rest, but the discontinuity gives rise to a shock and rarefaction waves propagating to the left and right, respectively. Therefore, in despite of its apparent one-dimensional simplicity, the shock tube case involves dynamics of a thermal, intrinsically compressible fluid flow. Namely, a shock wave increases the temperature of the medium through which it is traveling and, consequently, the speed of sound increases. Thus, information travels faster and this causes the signals in the region behind a shock to have a tendency to approach the shock. We simulate the problem using the fourth-order, thermal LBE together with the D2V37 velocity set (see Fig. 9 as well as Refs. 20 and 29).

The fluid dynamics related to the Sod shock tube case is described by non-linear hyperbolic equations and their numerical solution continues to be a challenge, especially with a large density step. Numerous computational methods have been proposed for the solution 


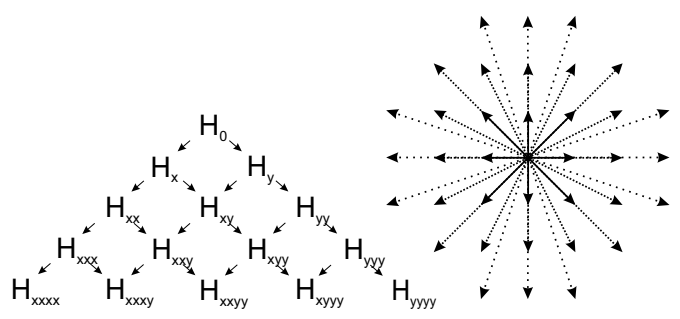

(a) (b)

FIG. 9. Fourth-order Hermitian basis of the subspace $\mathcal{H}_{q=15}$ and the conforming D2V37 discrete velocity set.

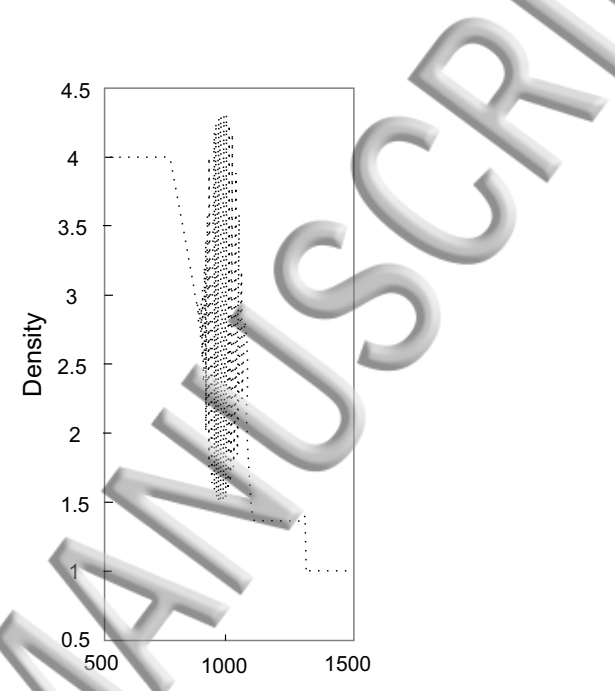

FIG. 10. Density profile in a Sod shock tube after 200 time steps using the standard LBGK scheme with $\tau^{*}=0.6$. The simulation is already unstable: the high-frequency oscillations are evident.

of the hyperbolic equations in the case of inviscid, non heat-conducting fluids. Sod ${ }^{44}$ compared many of these methods: he observed that most of the methods produce unphysical oscillations while the remaining methods have difficulties in reproducing the sharp discontinuities.

The Sod shock tube problem was also treated by Ansumali and Karlin ${ }^{45}$ using the entropic LBGK scheme with an initial density ratio of 2:1. Although the entropic scheme was more stable in simulations than the standard LBGK scheme, notable smoothing of the density and velocity profiles was observed. In fact, the entropic scheme used by Ansumali and Karlin $^{45}$ was intended for isothermal flows. Subsequently, Chikatamarla and Karlin ${ }^{43}$ used a one-dimensional, 5-velocity thermal entropic scheme for simulating a shock tube with a 3:1 density step: the sharp density profiles were now reproduced with a better accuracy; no results were presented for the temperature and velocity profiles. Philippi et al. ${ }^{46}$ simulated the shock tube problem using a 2D thermal LBE with 81 discrete velocities. After 2000 time steps, they observed no oscillations or smoothing with an initial density ratio of 4:1

Here the shock tube problem is simulated using D2V37 with three different LB schemes: the standard LBGK, LBGK supplemented with the original regularization, and LBGK supplemented with the new, refined regularization. Simulations are started with a density discontinuity at the middle of the domain, $x=1000$ (the width of the domain is 2000 lattice spacings), in such a manner that $\varrho=\varrho_{L}=4$ for $x<1000$ and $\varrho=\varrho_{R}=1$ for $x \geq 1000$ (the initial density ratio is thus 4:1). Several dimensionless relaxation times, $\tau^{*}=\tau / \Delta t$, were tested. Figure 10 shows the density profile for the standard LBGK scheme with $\tau^{*}=0.6$ after 200 time steps. High frequency oscillations are evident and the simulation is obviously compromised due to stability issues. Figures 11-13 compare the results obtained 


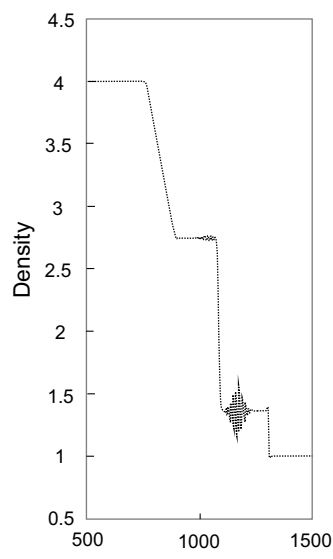

(a)

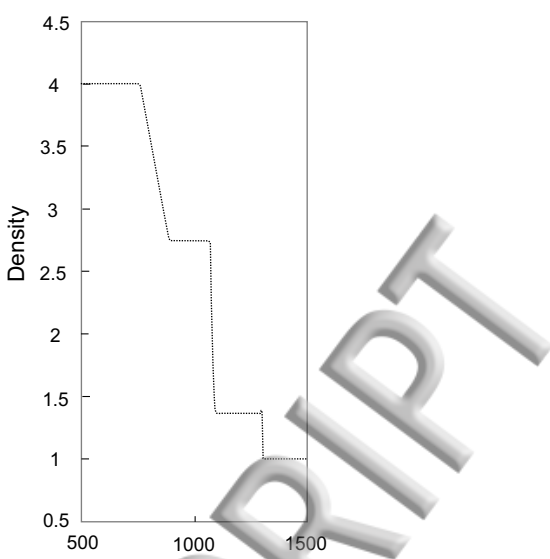

(b)

FIG. 11. Density profile in a Sod Shock tube after 200 time steps using the (a) original regularization and (b) refined regularization.

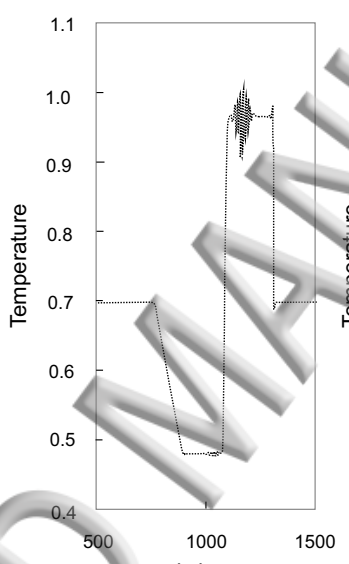

(a)

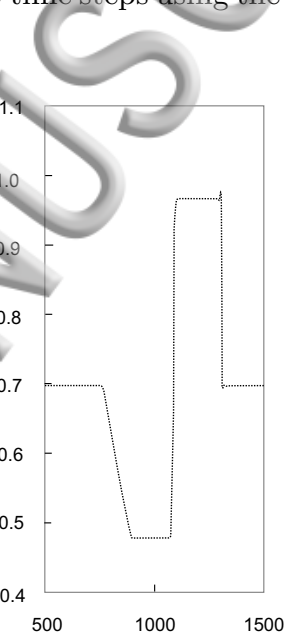

(b)

FIG. 12. Temperature profile in a Sod Shock tube after 200 time steps using the (a) original regularization and (b) refined regularization.

with the original and refined regularization procedures. Simulation results clearly show the stabilizing effect of the new regularization procedure when compared with the original one.

\section{CONCLUSIONS}

We have considered LBEs as discrete counterparts to continuous kinetic model equations projected onto a given subspace. The corresponding Hermitian basis, a finite set of Hermite polynomials, can be systematically linked to discrete velocity sets suitable for simulating a specific flow problem. In other words, LBEs with increasing order of approximation to a given kinetic model can be rigorously derived by adopting increasingly larger sets of Hermite polynomials leading, ultimately, to larger discrete velocity sets.

Accordingly, Hermite polynomial expansions of the distribution functions have been here strictly utilized. The important consequence of this systematic approach is that moment space representations of the distributions are immediately available (defined by the coefficients of the expansion). Alternative, particular moment space representations can be constructed by resorting to, e.g., the Gram-Schmidt orthogonalization procedure. Such 


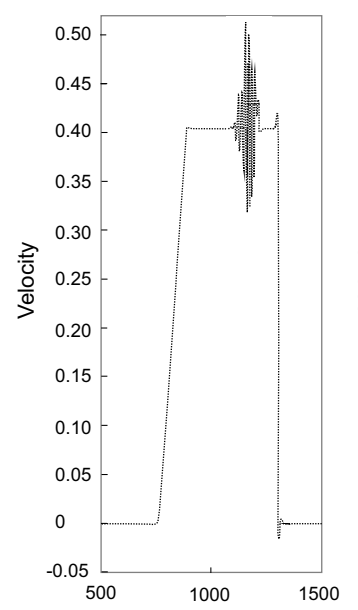

(a)

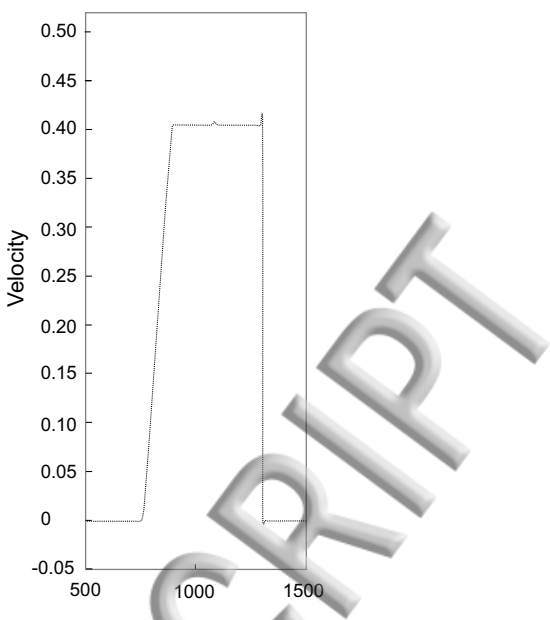

(b)

FIG. 13. Velocity profile in a Sod Shock tube after 200 time steps using the (a) original regularization and (b) refined regularization.

an approach, however, does not provide a uniform or generic solution for improving the stability of LBEs. Constructing tailored moment spaces, that conform with large discrete velocity sets, is problematic especially because the choice of moments does influence the stability properties of a LB scheme: a poor choice of moments can lead to suboptimal stability ranges.

Regularization, a procedure for filtering the high-order non-equilibrium moments that do not fit into the Hermitian representation, or are beyond the macroscopic equations targeted, was here considered as a general tool for improving stability of LB schemes particularly in conjunction with high-order LBEs, large discrete velocity sets, and in the case of thermal flows. A new, refined regularization procedure was proposed, where only the diffusive parts of non-equilibrium moments are filtered after a given rank: the diffusive parts are defined as peculiar or cental Hermite moments of the distributions. In the case of isothermal and thermal fluid flows, the diffusive parts are filtered after rank 2 and 3, respectively.

Filtering of diffusive parts only, while the advective parts are retained in the expansions, leads to specific recurrence relations and to the expression of high-order non-equilibrium moments in terms of low-order ones. These recurrence relations provide a convenient way to implement the proposed regularization, and incur only a minor overhead in computational requirements. Using a linear stability analysis, it can be shown that in the special case of second-order LBEs, used together with the D2Q9 and D3Q27 velocity sets, the particular expressions emerging from the general recurrence relations here presented indeed lead to improved stability ranges. Here numerical experiments with the perturbed double periodic shear layer flow (isothermal) and Sod shock tube problem, involving a compressible and thermal fluid flow, further demonstrate that the new, refined regularization procedure improves the stability of LB schemes without distinct optimization steps or ad-hoc assumptions.

Here the main focus has been on theoretical aspects of the regularization procedure, and its application to LBEs in general. Finally we want to emphasize that although here the treatment was limited to the kinetic BGK model equation, application of the regularization to other kinetic models is certainly possible, including models for multiphase and non-ideal multicomponent systems. 


\section{ACKNOWLEDGMENTS}

The financial support of Petrobras and $\mathrm{CNPq}$ is gratefully acknowledged. Furthermore, we acknowledge the financial support from the European Community's Seventh Framework programme NMP.2013.1.4-1 under Grant Agreement no. 604005. We are also thankful for the computational resources provided by CSC IT Center for Science Ltd (Finland).

\section{Appendix: LB schemes for the D2Q9 velocity set}

For a given discrete velocity set, let $\boldsymbol{e}_{i}$ denote dimensionless lattice vectors (with strictly integer components on square and cubic lattices). These lattice vectors are related to the dimensionless velocity vectors, i.e. $\boldsymbol{\xi}_{i}=a_{s} \boldsymbol{e}_{i}$, where $a_{s}$ is the scaling factor of a discrete velocity set (e.g. $a_{s}=\sqrt{3}$ for D2Q9, D3Q15, D3Q19, and D3Q27). We then specify the first few Hermite polynomial tensors in terms of the lattice vectors:

$$
\begin{aligned}
H_{0}\left(\boldsymbol{e}_{i}\right):= & \frac{1}{a_{s}^{0}} H_{0}\left(\boldsymbol{\xi}_{i}\right)=1, \\
H_{\alpha}\left(\boldsymbol{e}_{i}\right):= & \frac{1}{a_{s}^{1}} H_{\alpha}\left(\boldsymbol{\xi}_{i}\right)=e_{i, \alpha}, \\
H_{\alpha \beta}\left(\boldsymbol{e}_{i}\right):= & \frac{1}{a_{s}^{2}} H_{\alpha \beta}\left(\boldsymbol{\xi}_{i}\right)=e_{i, \alpha} e_{i, \beta}-\frac{1}{a_{s}^{2}} \delta_{\alpha \beta}, \\
H_{\alpha \beta \gamma}\left(\boldsymbol{e}_{i}\right):= & \frac{1}{a_{s}^{3}} H_{\alpha \beta \gamma}\left(\boldsymbol{\xi}_{i}\right)=e_{i, \alpha} e_{i, \beta} e_{i, \gamma} \\
& -\frac{1}{a_{s}^{2}}\left(e_{i, \alpha} \delta_{\beta \gamma}+e_{i, \beta} \delta_{\alpha \gamma}+e_{i, \gamma} \delta_{\alpha \beta}\right), \\
H_{\alpha \beta \gamma \delta}\left(\boldsymbol{e}_{i}\right):= & \frac{1}{a_{s}^{4}} H_{\alpha \beta \gamma \delta}\left(\boldsymbol{\xi}_{i}\right)=e_{i, \alpha} e_{i, \beta} e_{i, \gamma} e_{i, \delta} \\
& -\frac{1}{a_{s}^{2}}\left(e_{i, \alpha} e_{i, \beta} \delta_{\gamma \delta}+e_{i, \alpha} e_{i, \gamma} \delta_{\beta \delta}+e_{i, \alpha} e_{i, \delta} \delta_{\beta \gamma}\right. \\
& \left.+e_{i, \beta} e_{i, \gamma} \delta_{\alpha \delta}+e_{i, \beta} e_{i, \delta} \delta_{\alpha \gamma}+e_{i, \gamma} e_{i, \delta} \delta_{\alpha \beta}\right) \\
& +\frac{1}{a_{s}^{4}}\left(\delta_{\alpha \beta} \delta_{\gamma \delta}+\delta_{\alpha \gamma} \delta_{\beta \delta}+\delta_{\alpha \delta} \delta_{\beta \gamma}\right) .
\end{aligned}
$$

Moreover, using the above definitions, we specify kinetic projectors as

$$
K^{(j, k, l)}\left(\boldsymbol{e}_{i}\right):=w_{i} \frac{a_{s}^{2(j+k+l)}}{j ! k ! l !} H_{\alpha}^{(j, k, l)}\left(\boldsymbol{e}_{i}\right) .
$$

Below all dimensionless moments of the (dimensionless) distribution function are computed using $\boldsymbol{e}_{i}$, not $\boldsymbol{\xi}_{i}$. In particular,

$$
\rho u_{\alpha}=\sum_{i} e_{i, \alpha} f_{i}, \quad \tau_{\alpha \beta}=\sum_{i} e_{i, \alpha} e_{i, \alpha}\left(f_{i}-f_{i}^{e q}\right),
$$

where $\boldsymbol{\tau}$ is evaluated after streaming, i.e. before collision. A finite Hermite expansion of the distribution can now be written in a very simple form,

$$
f_{i}:=\sum_{H \in \mathcal{H}_{q}} a^{(j, k, l)} K^{(j, k, l)}\left(\boldsymbol{e}_{i}\right)
$$

Utilizing these notations, specific LB schemes together with the D2Q9 velocity set are listed in Table I. 
TABLE I. List of LB schemes for the D2Q9 discrete velocity set: $f_{i}^{\text {out }}=f_{i}^{e q}+\left(1-\frac{\delta t}{\tau}\right) f_{i}^{n e q}$.

\begin{tabular}{|c|c|c|}
\hline Hermitian representation & Distributions & Note \\
\hline $\mathrm{H}_{\mathrm{xx}}$ & $\begin{array}{l}f_{i}^{e q}=w_{i} \rho\left[1+a_{s}^{2} u_{\alpha} e_{i, \alpha}+\frac{a_{s}^{4}}{2} u_{\alpha} u_{\beta}\left(e_{i, \alpha} e_{i, \beta}-\frac{1}{a_{s}^{2}} \delta_{\alpha \beta}\right)\right] \\
f_{i}^{n e q}=f_{i}-f_{i}^{e q}(\mathrm{BGK})\end{array}$ & \multirow{2}{*}{$\begin{array}{l}\text { The most widely used LBE both in } \\
2 \mathrm{D} \text { and } 3 \mathrm{D} \text { : a } 2 \text { nd-order approxima- } \\
\text { tion to the MB equilibrium distri- } \\
\text { bution and a BGK relaxation term } \\
\text { The standard regularization: Latt } \\
\text { and Chopard }{ }^{18} \text { reported an increase } \\
\text { in the stability range by a factor } \\
7 \text { in the lid-driven cavity problem } \\
\text { Comparison with entropic stabilizer } \\
\text { by Mattila et al. }{ }^{19} \text {. }\end{array}$} \\
\hline & $\begin{array}{l}f_{i}^{e q}=w_{i} \rho\left[1+a_{s}^{2} u_{\alpha} e_{i, \alpha}+\frac{a_{s}^{4}}{2} u_{\alpha} u_{\beta}(e\right. \\
f_{i}^{n e q}=w_{i} \frac{a_{s}^{4}}{2} \tau_{\alpha \beta}\left(e_{i, \alpha} e_{i, \beta}-\frac{1}{a_{s}^{2}} \delta_{\alpha \beta}\right)\end{array}$ & \\
\hline & $\begin{array}{l}\left.+a_{s}^{2} u_{\alpha} e_{i, \alpha}+\frac{a_{s}^{4}}{2} u_{\alpha} u_{\beta}\left(e_{i, \alpha} e_{i, \beta}-\frac{1}{a_{s}^{2}} \delta_{\alpha \beta}\right)\right] \\
\left.\left(e_{i, x}^{2}-\frac{1}{a_{s}^{2}}\right) e_{i, y}+\frac{a_{s}^{6}}{2} u_{y}^{2} u_{x}\left(e_{i, y}^{2}-\frac{1}{a_{s}^{2}}\right) e_{i, x}\right] \\
f_{i}^{e q}(\mathrm{BGK})\end{array}$ & $\begin{array}{l}\text { This LBE was used by Siebert } \\
\text { et al. }{ }^{13} \text { showing improved stabil- } \\
\text { ity results when compared to the } \\
\text { MRT scheme used by Lallemand } \\
\text { and Luo }{ }^{47} \text {. }\end{array}$ \\
\hline $\mathrm{H}_{\mathrm{xxy}}$ & $\begin{array}{l}\left.+a_{s}^{2} u_{\alpha} e_{i, \alpha}+\frac{a_{s}^{4}}{2} u_{\alpha} u_{\beta}\left(e_{i, \alpha} e_{i, \beta}-\frac{1}{a_{s}^{2}} \delta_{\alpha \beta}\right)\right] \\
\left(e_{i, x}^{2}-\frac{1}{a_{s}^{2}}\right) e_{i, y}+\frac{a_{s}^{6}}{2} u_{y}^{2} u_{x}\left(e_{i, y}^{2}-\frac{1}{a_{s}^{2}}\right) e_{i, x} \\
\left.\left(e_{i, x}^{2}-\frac{1}{a_{s}^{2}}\right)\left(e_{i, y}^{2}-\frac{1}{a_{s}^{2}}\right)\right]\end{array}$ & $\begin{array}{l}\text { The BGK relaxation term with } \\
\text { a full Hermitian expansion of the } \\
\text { equilibrium distribution. Used in } \\
\text { Sec.V A for the simulation of per- } \\
\text { turbed double periodic shear layer } \\
\text { flow. }\end{array}$ \\
\hline & $\begin{aligned} f_{i}^{e q} & =w_{i} \rho\left[1+a_{s}^{2} u_{\alpha} e_{i, \alpha}+\frac{a_{s}^{4}}{2} u_{\alpha} u_{\beta}\left(e_{i, \alpha} e_{i, \beta}-\frac{1}{a_{s}^{2}} \delta_{\alpha \beta}\right)\right] \\
& +\frac{a_{s}^{6}}{2} u_{x}^{2} u_{y}\left(e_{i, x}^{2}-\frac{1}{a_{s}^{2}}\right) e_{i, y}+\frac{a_{s}^{6}}{2} u_{y}^{2} u_{x}\left(e_{i, y}^{2}-\frac{1}{a_{s}^{2}}\right) e_{i, x} \\
& \left.+\frac{a_{s}^{8}}{4} u_{x}^{2} u_{y}^{2}\left(e_{i, x}^{2}-\frac{1}{a_{s}^{2}}\right)\left(e_{i, y}^{2}-\frac{1}{a_{s}^{2}}\right)\right] \\
f_{i}^{n e q} & =w_{i} \frac{a_{s}^{4}}{2} \tau_{\alpha \beta}\left(e_{i, \alpha} e_{i, \beta}-\frac{1}{a_{s}^{2}} \delta_{\alpha \beta}\right)\end{aligned}$ & $\begin{array}{l}\text { The standard regularization scheme } \\
\text { with a full Hermitian expansion of } \\
\text { the equilibrium distribution, but } \\
\text { only a 2nd-order Hermitian expan- } \\
\text { sion for the non-equilibrium distri- } \\
\text { bution. Used in Sec.V A for the } \\
\text { simulation of perturbed double pe- } \\
\text { riodic shear layer flow. }\end{array}$ \\
\hline $\mathrm{H}_{\mathrm{xxyy}}$ & $\begin{aligned} & f_{i}^{e q}=w_{i} \rho\left[1+a_{s}^{2} u_{\alpha} e_{i, \alpha}+\frac{a_{s}^{4}}{2} u_{\alpha} u_{\beta}\left(e_{i, \alpha} e_{i, \beta}-\frac{1}{a_{s}^{2}} \delta_{\alpha \beta}\right)\right] \\
&+\frac{a_{s}^{6}}{2} u_{x}^{2} u_{y}\left(e_{i, x}^{2}-\frac{1}{a_{s}^{2}}\right) e_{i, y}+\frac{a_{s}^{6}}{2} u_{y}^{2} u_{x}\left(e_{i, y}^{2}-\frac{1}{a_{s}^{2}}\right) e_{i, x} \\
&\left.+\frac{a_{s}^{8}}{4} u_{x}^{2} u_{y}^{2}\left(e_{i, x}^{2}-\frac{1}{a_{s}^{2}}\right)\left(e_{i, y}^{2}-\frac{1}{a_{s}^{2}}\right)\right] \\
& f_{i}^{n e q}=w_{i}\left[\frac{a_{s}^{4}}{2} \tau_{\alpha \beta}\left(e_{i, \alpha} e_{i, \beta}-\frac{1}{a_{s}^{2}} \delta_{\alpha \beta}\right)\right. \\
&+\frac{a_{s}^{6}}{2}\left(u_{y} \tau_{x x}+2 u_{x} \tau_{x y}\right)\left(e_{i, x}^{2}-\frac{1}{a_{s}^{2}}\right) e_{i, y} \\
&+\frac{a_{s}^{6}}{2}\left(u_{x} \tau_{y y}+2 u_{y} \tau_{x y}\right)\left(e_{i, y}^{2}-\frac{1}{a_{s}^{2}}\right) e_{i, x} \\
&\left.+\frac{a_{s}^{8}}{4}\left(u_{y}^{2} \tau_{x x}+4 u_{x} u_{y} \tau_{x y}+u_{x}^{2} \tau_{y y}\right)\left(e_{i, x}^{2}-\frac{1}{a_{s}^{2}}\right)\left(e_{i, y}^{2}-\frac{1}{a_{s}^{2}}\right)\right]\end{aligned}$ & $\begin{array}{l}\text { A refined regularization scheme } \\
\text { with a full Hermitian expansion } \\
\text { of both the equilibrium and non- } \\
\text { equilibrium distribution. The re- } \\
\text { currence relations are used for the } \\
\text { 3rd- and 4th-order non-equilibrium } \\
\text { moments. Used in Sec.V A for the } \\
\text { simulation of perturbed double pe- } \\
\text { riodic shear layer flow. }\end{array}$ \\
\hline
\end{tabular}

${ }^{1}$ G. R. McNamara and G. Zanetti, "Use of the boltzmann equation to simulate lattice-gas automata," Physical Review Letters 61, 2332-2335 (1988). 
${ }^{2}$ F. J. Higuera, S. Succi, and R. Benzi, "Lattice Gas Dynamics with Enhanced Collisions," Europhysics Letters (EPL) 9, 345-349 (1989).

${ }^{3}$ R. Brownlee, J. Levesley, D. Packwood, and A. Gorban, "Add-ons for lattice boltzmann methods: Regularization, filtering and limiters," in Novel Trends in Lattice-Boltzmann Methods, Progress in Computational Physics, Vol. 3, edited by M. Ehrhardt (Bentham Science Publishers, Sharjah, U.A.E., 2013) Chap. 2, p. 31.

${ }^{4}$ D. Golbert, P. Blanco, A. Clausse, and R. Feijóo, "On the search of more stable second-order latticeBoltzmann schemes in confined flows," Journal of Computational Physics 294, 605-618 (2015).

${ }^{5}$ D. D'Humieres, "Generalized Lattice-Boltzmann Equations," in Rarefied Gas Dynamics: Theory and Simulations, Vol. 159 (American Institute of Aeronautics and Astronautics, Washington DC, 1994) pp. 450-458.

${ }^{6}$ D. D'Humières, I. Ginzburg, M. Krafczyk, P. Lallemand, and L.-S. Luo, "Multiple-relaxation-time lattice Boltzmann models in three dimensions." Philosophical transactions. Series A, Mathematical, physical, and engineering sciences 360, 437-51 (2002).

${ }^{7}$ P. J. Dellar, "Incompressible limits of lattice Boltzmann equations using multiple relaxation times," Journal of Computational Physics 190, 351-370 (2003).

${ }^{8}$ P. Lallemand and L.-S. Luo, "Theory of the lattice Boltzmann method: Acoustic and thermal properties in two and three dimensions," Physical Review E 68, 036706 (2003).

${ }^{9} \mathrm{H}$. Xu and P. Sagaut, "Optimal low-dispersion low-dissipation LBM schemes for computational aeroacoustics," Journal of Computational Physics 230, 5353-5382 (2011).

${ }^{10}$ I. V. Karlin, A. Ferrante, and H. C. Öttinger, "Perfect entropy functions of the Lattice Boltzmann method," Europhysics Letters (EPL) 47, 182-188 (1999).

${ }^{11}$ P. Bhatnagar, E. Gross, and M. Krook, "A Model for Collision Processes in Gases. I. Small Amplitude Processes in Charged and Neutral One-Component Systems," Physical Review 94, 511-525 (1954).

${ }^{12}$ I. V. Karlin, F. Bösch, and S. S. Chikatamarla, "Gibbs' principle for the lattice-kinetic theory of fluid dynamics," Physical Review E 90, 031302 (2014).

${ }^{13}$ D. N. Siebert, L. A. Hegele Júnior, and P. C. Philippi, "Lattice Boltzmann equation linear stability analysis: Thermal and athermal models," Physical Review E 77, 026707 (2008).

${ }^{14}$ R. Adhikari and S. Succi, "Duality in matrix lattice boltzmann models," Physical Review E 78, 066701 (2008).

15 A. J. C. Ladd, "Numerical simulations of particulate suspensions via a discretized Boltzmann equation. Part 1. Theoretical foundation," Journal of Fluid Mechanics 271, 285 (1994).

${ }^{16} \mathrm{H}$. Chen, R. Zhang, I. Staroselsky, and M. Jhon, "Recovery of full rotational invariance in lattice Boltzmann formulations for high Knudsen number flows," Physica A: Statistical Mechanics and its Applications 362, 125-131 (2006).

${ }^{17}$ R. Zhang, X. Shan, and H. Chen, "Effieient kinetic method for fluid simulation beyond the Navier-Stokes equation," Physical Review E 74, 046703 (2006).

${ }^{18}$ J. Latt and B. Chopard, "Lattice Boltzmann method with regularized pre-collision distribution functions," Mathematics and Computers in Simulation 72, 165-168 (2006).

${ }^{19}$ K. K. Mattila, L. A. Hegele, and P. C. Philippi, "Investigation of an entropic stabilizer for the latticeBoltzmann method"' Physical Review E 91, 063010 (2015).

${ }^{20} \mathrm{X}$. Shan and H. Chen, "A general multiple-relaxation-time Boltzmann collision model," International Journal of Modern Physics C 18, 635-643 (2007).

${ }^{21} \mathrm{H}$. Chen and X. Shan, "Fundamental conditions for N-th-order accurate lattice Boltzmann models," Physica D: Nonlinear Phenomena 237, 2003-2008 (2008).

${ }^{22} \mathrm{O}$. Malaspinas, "Increasing stability and accuracy of the lattice Boltzmann scheme: recursivity and regularization," arXiv:1505.06900v1 [physics.flu-dyn] 26 May 2015 , 1-31 (2015).

${ }^{23}$ A. Montessori, P. Prestininzi, M. La Rocca, and S. Succi, "Lattice Boltzmann approach for complex nonequilibrium flows," Physical Review E 92, 043308 (2015).

${ }^{24}$ F. Dubois, T. Février, and B. Graille, "Lattice Boltzmann Schemes with Relative Velocities," Communications in Computational Physics 17, 1088-1112 (2015).

${ }^{25}$ F. Dubois, T. Février, and B. Graille, "On the stability of a relative velocity lattice Boltzmann scheme for compressible NavierStokes equations," Comptes Rendus Mécanique 343, 599-610 (2015).

${ }^{26}$ J. C. Maxwell, "On the dynamical theory of gases," Philosophical transactions of the Royal Society 157, 49-88 (1867)

${ }^{27}$ L. Boltzmann, Vorlesungen uber Gas Theory, Part I (J.A. Barth, Leipzig, 1896).

${ }^{28}$ L. Boltzmann, Vorlesungen uber Gas Theory, Part II (J.A. Barth, Leipzig, 1898) p. 290.

${ }^{29}$ P. C. Philippi, L. A. Hegele Júnior, L. O. E. dos Santos, and R. Surmas, "From the continuous to the lattice Boltzmann equation: The discretization problem and thermal models," Physical Review E $\mathbf{7 3}$, $056702(2006)$.

${ }^{30}$ D. Siebert, L. A. Hegele Júnior, R. Surmas, L. dos Santos, and P. Philippi, "Thermal lattice Boltzmann in two dimensions," International Journal of Modern Physics C 18, 546-555 (2007).

${ }^{31}$ X. Shan, "General solution of lattices for Cartesian lattice Bhatanagar-Gross-Krook models," Physical Review E 81, 036702 (2010).

${ }^{32}$ K. Mattila, L. Hegele Jr, and P. Philippi, "High-Accuracy Approximation of High-Rank Derivatives: Isotropic Finite Differences Based on Lattice-Boltzmann Stencils," The Scientific World Journal 2014 142907 (2014). 
${ }^{33}$ T. Ruggeri, "Galilean invariance and entropy principle for systems of balance laws - The structure of extended thermodynamics," Continuum Mechanics and Thermodynamics 1, 3-20 (1989).

${ }^{34}$ M. Torrilhon, J. Au, and H. Struchtrup, "Explicit fluxes and productions for large systems of the moment method based on extended thermodynamics," Continuum Mechanics and Thermodynamics 15, 97-111 (2003).

${ }^{35}$ S. S. Chikatamarla and I. V. Karlin, "Lattices for the lattice Boltzmann method," Physical Review E 79, 046701 (2009).

${ }^{36}$ P. J. Dellar, "Lattice Boltzmann algorithms without cubic defects in Galilean invariance on standard lattices," Journal of Computational Physics 259, 270-283 (2014).

${ }^{37}$ X. Shan, X.-F. Yuan, and H. Chen, "Kinetic theory representation of hydrodynamics: a way beyond the Navier-Stokes equation," Journal of Fluid Mechanics 550, 413-441 (2006).

${ }^{38}$ A. Montessori, G. Falcucci, P. Prestininzi, M. La Rocca, and S. Succi, "Regularized lattice BhatnagarGross-Krook model for two- and three-dimensional cavity flow simulations," Physical Review E 89, 053317 (2014).

${ }^{39}$ X.-D. Niu, S.-A. Hyodo, T. Munekata, and K. Suga, "Kinetic lattice boltzmann method for microscale gas flows: Issues on boundary condition, relaxation time, and regularization," Phys. Rev. E 76, 036711 (2007).

${ }^{40}$ D. Brown, "Performance of under-resolved two-dimensional incompressible flow simulations," Journal of Computational Physics 122, 165-183 (1995).

${ }^{41} \mathrm{M}$. Minion and D. Brown, "Performance of under-resolved two-dimensional incompressible flow simulations, ii," Journal of Computational Physics 138, 734-765 (1997).

${ }^{42}$ M. Reider and J. Sterling, "Accuracy of discrete-velocity bgk models for the simulation of the incompressible navier-stokes equations," Computers \& Fluids 24, 459-467 (1995).

${ }^{43}$ S. S. Chikatamarla and I. V. Karlin, "Entropy and Galilean Invariance of Lattice Boltzmann Theories," Physical Review Letters 97, 190601 (2006).

${ }^{44}$ G. A. Sod, "A survey of several finite difference methods for systems of nonlinear hyperbolic conservation laws," Journal of Computational Physics 27, 1-31 (1978).

${ }^{45}$ S. Ansumali and I. V. Karlin, "Stabilization of the lattice Boltzmann method by the H theorem: A numerical test," Physical Review E 62, 7999-8003 (2000).

${ }^{46}$ P. Philippi, L. Hegele Junior, R. Surmas, D. Siebert, and L. dos Santos, "From the Boltzmann to the lattice-Boltzmann equation: beyond BGK collision models," International Journal of Modern Physics C 18, 556-565 (2007).

${ }^{47}$ P. Lallemand and L.-S. Luo, "Theory of the lattice Boltzmann Method: Dispersion, Dissipation, Isotropy, Galilean Invariance, and Stability," Physical Reyiew E 61, 6546-6562 (2000). 


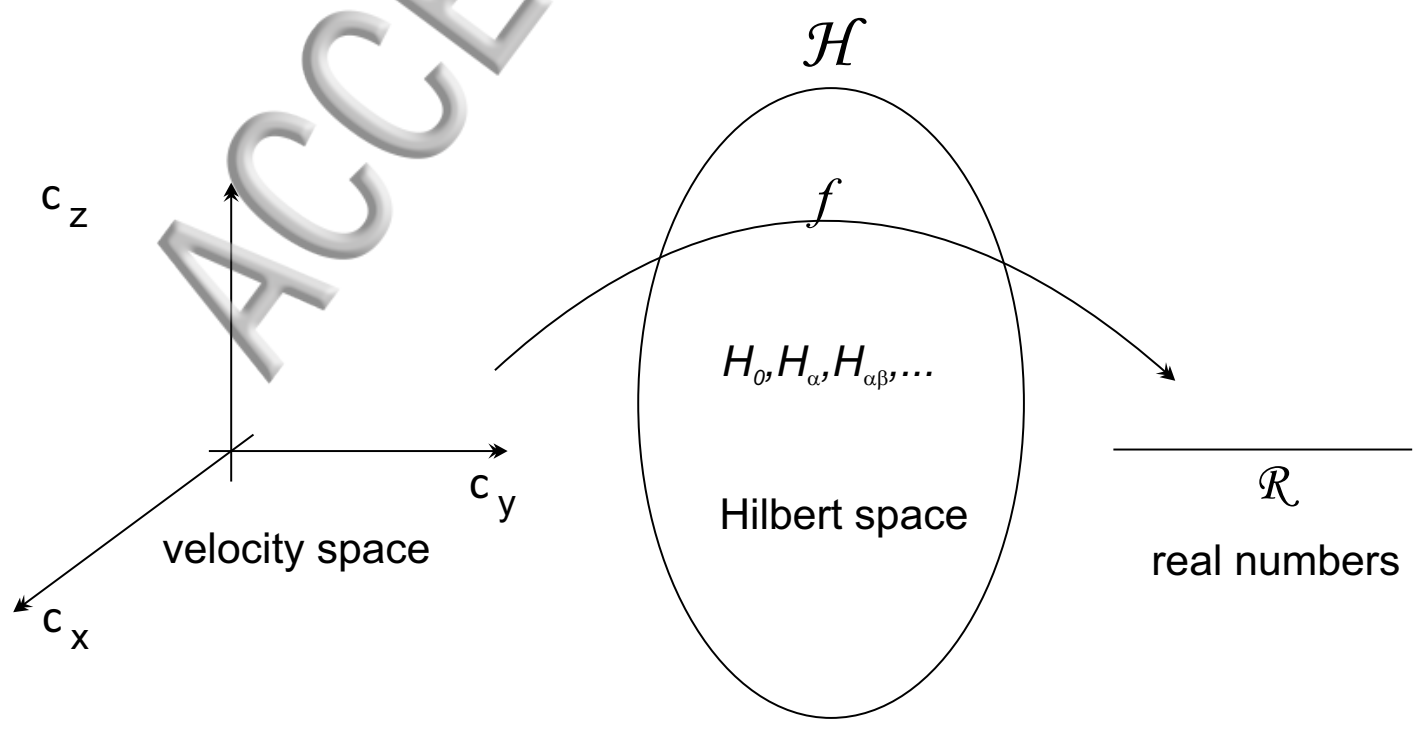




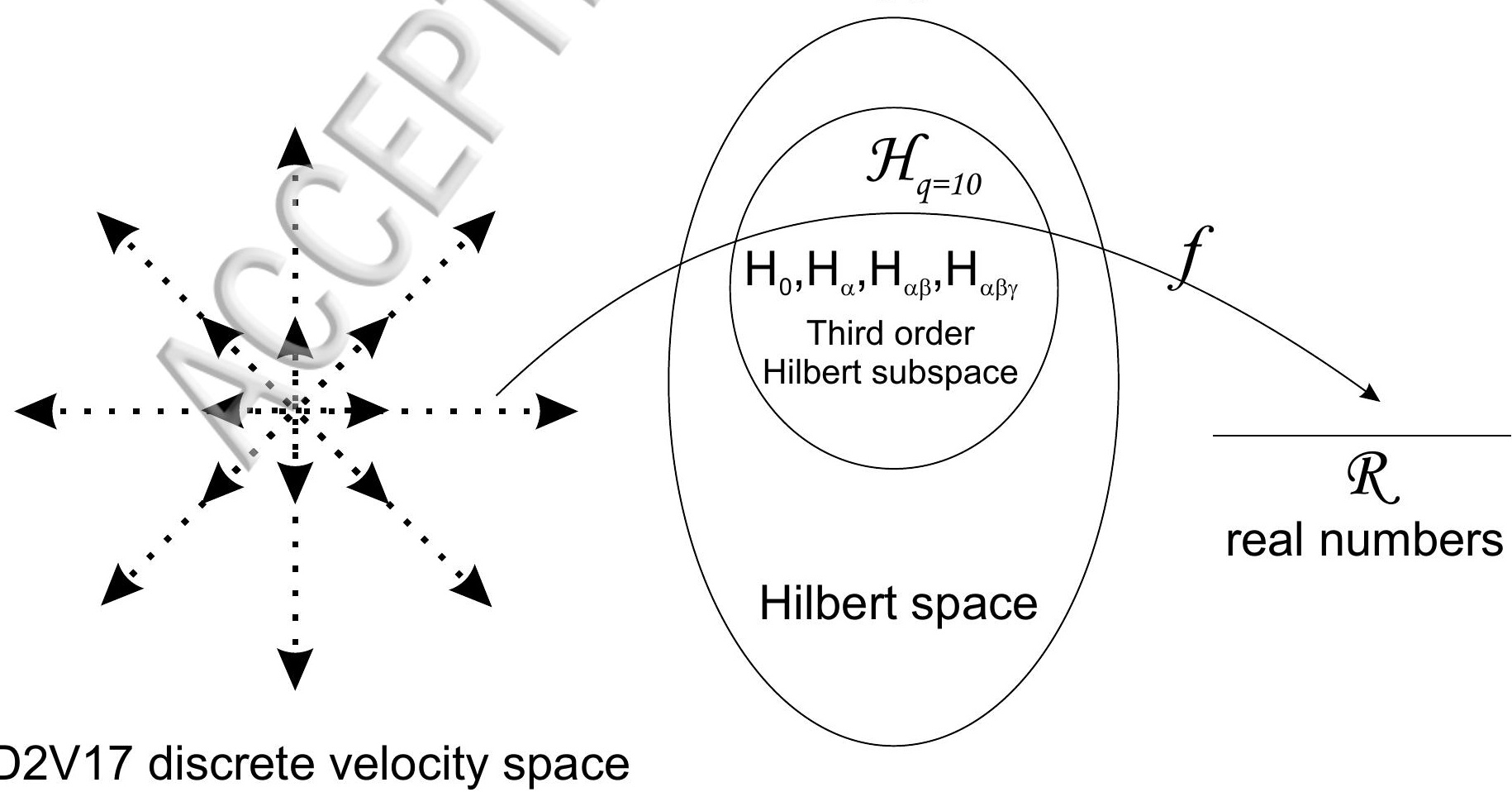




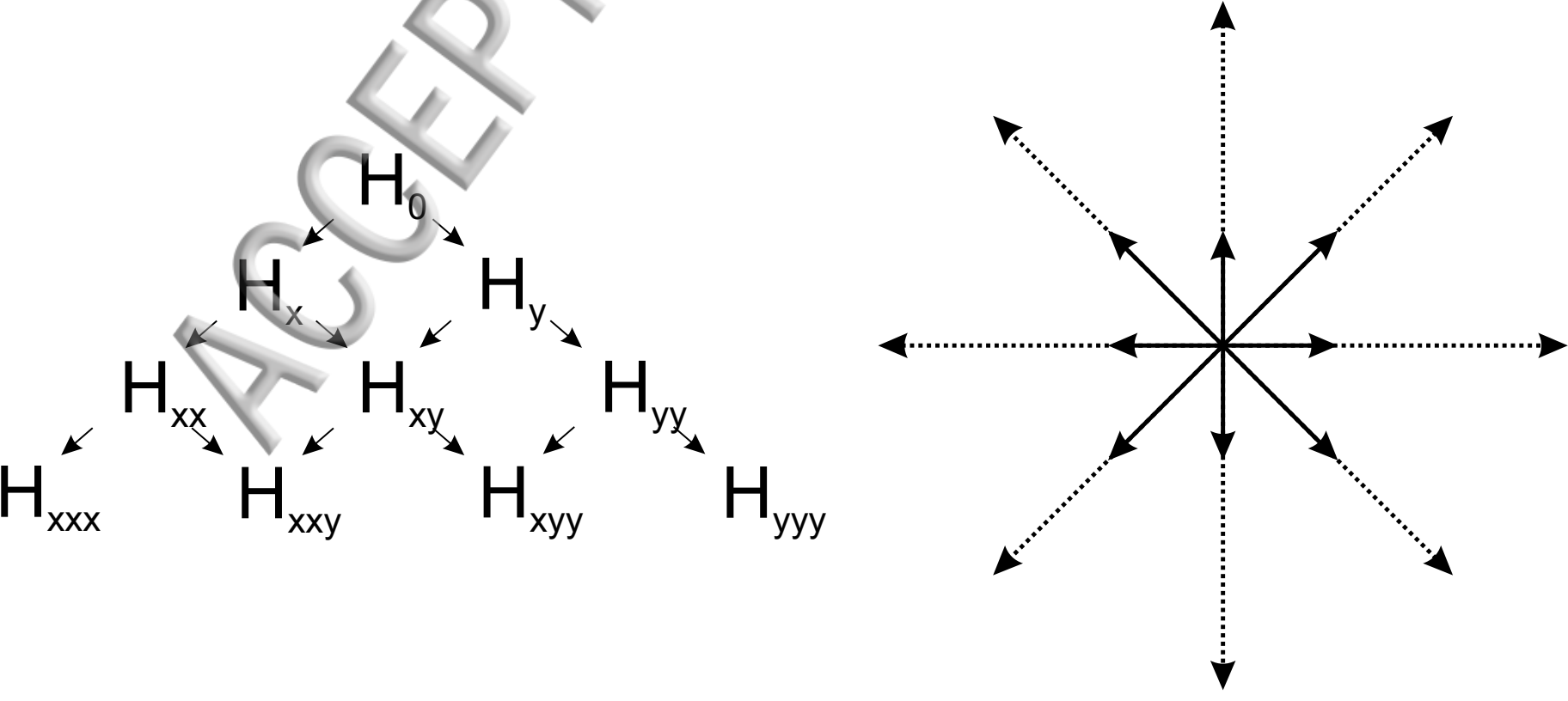

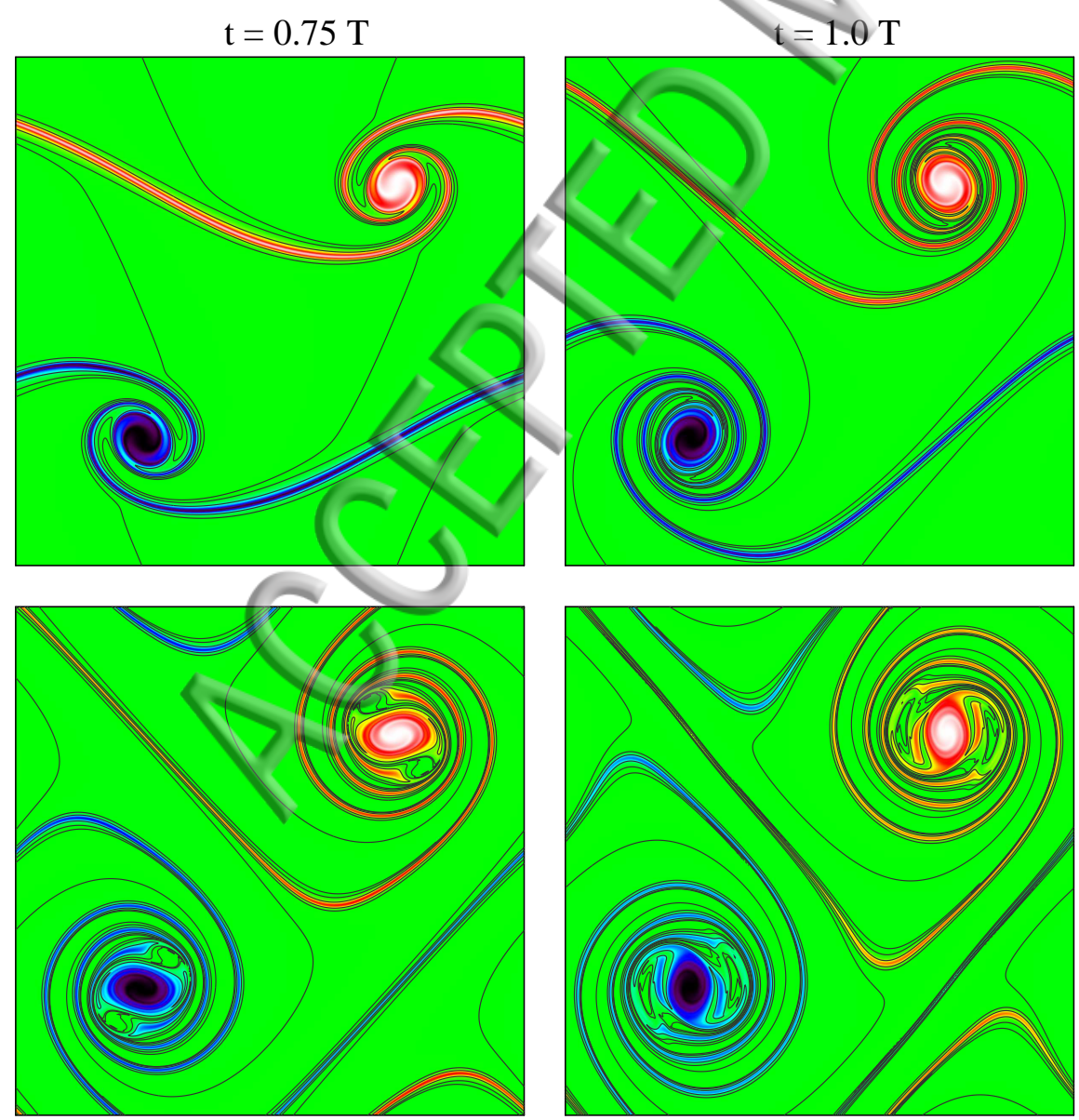

$$
\mathrm{t}=1.25 \mathrm{~T}
$$

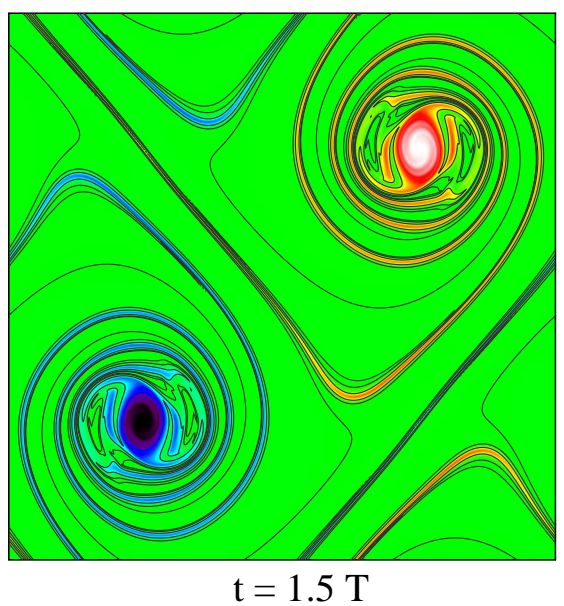




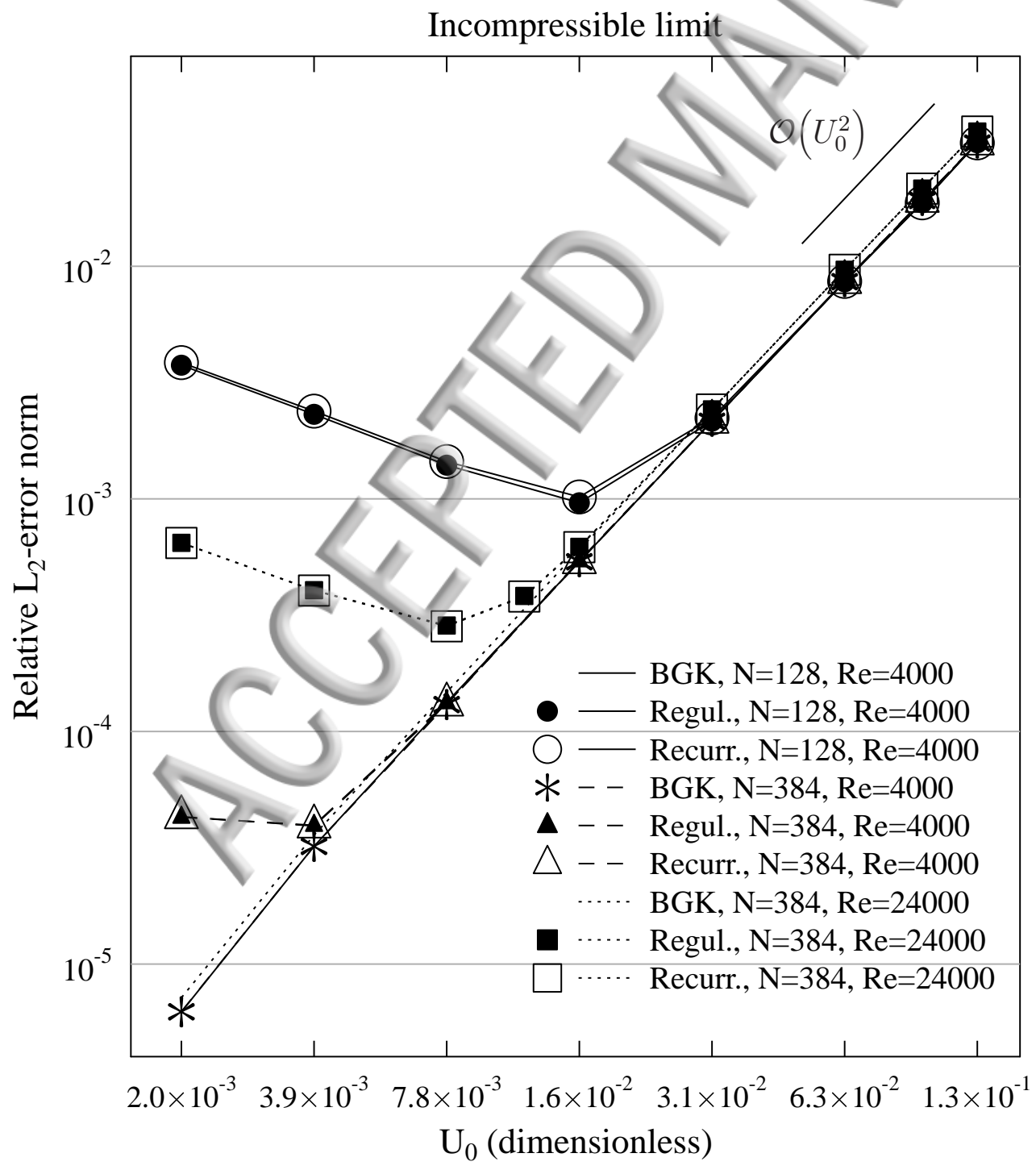


Stability map $(N=128)$

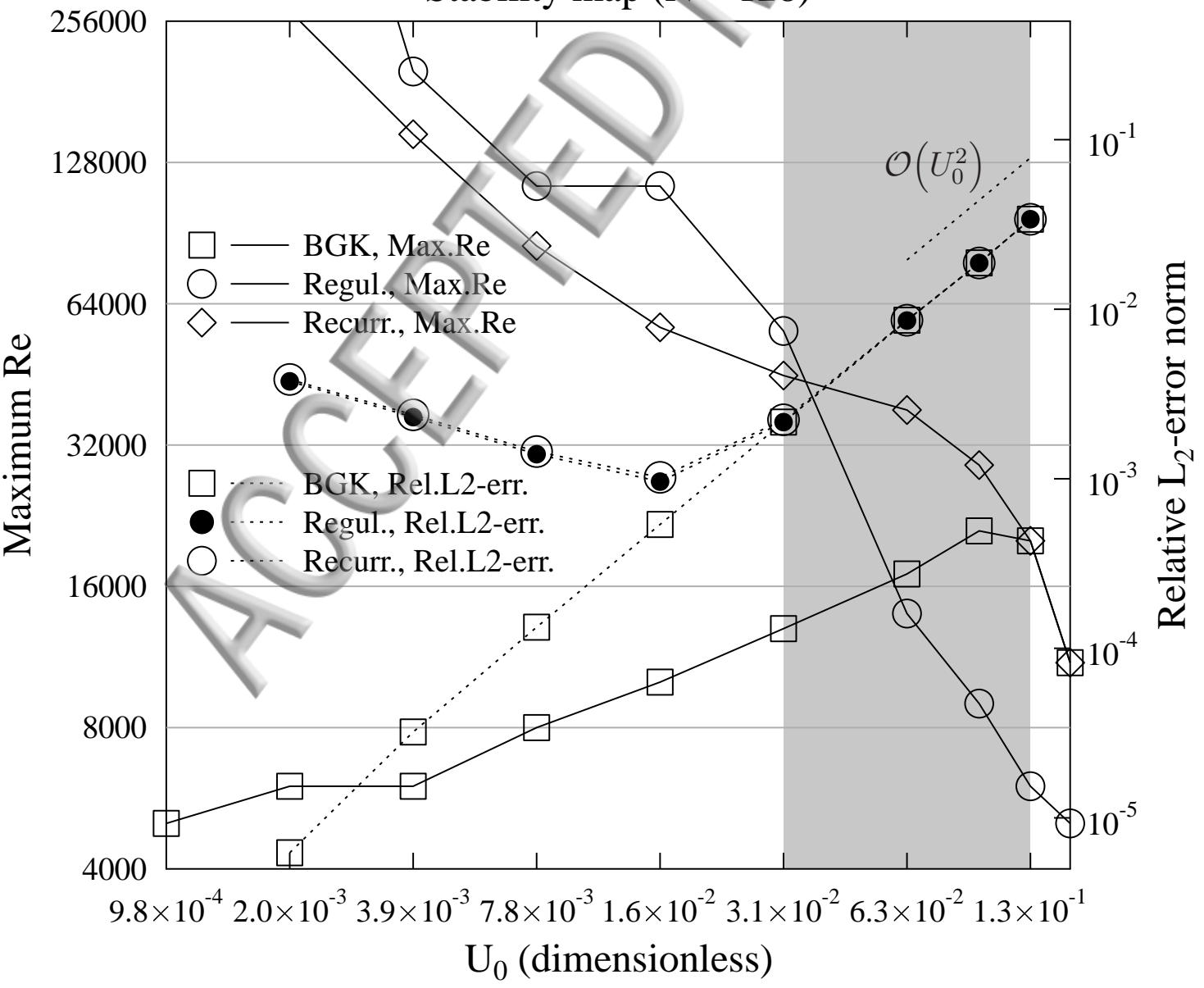



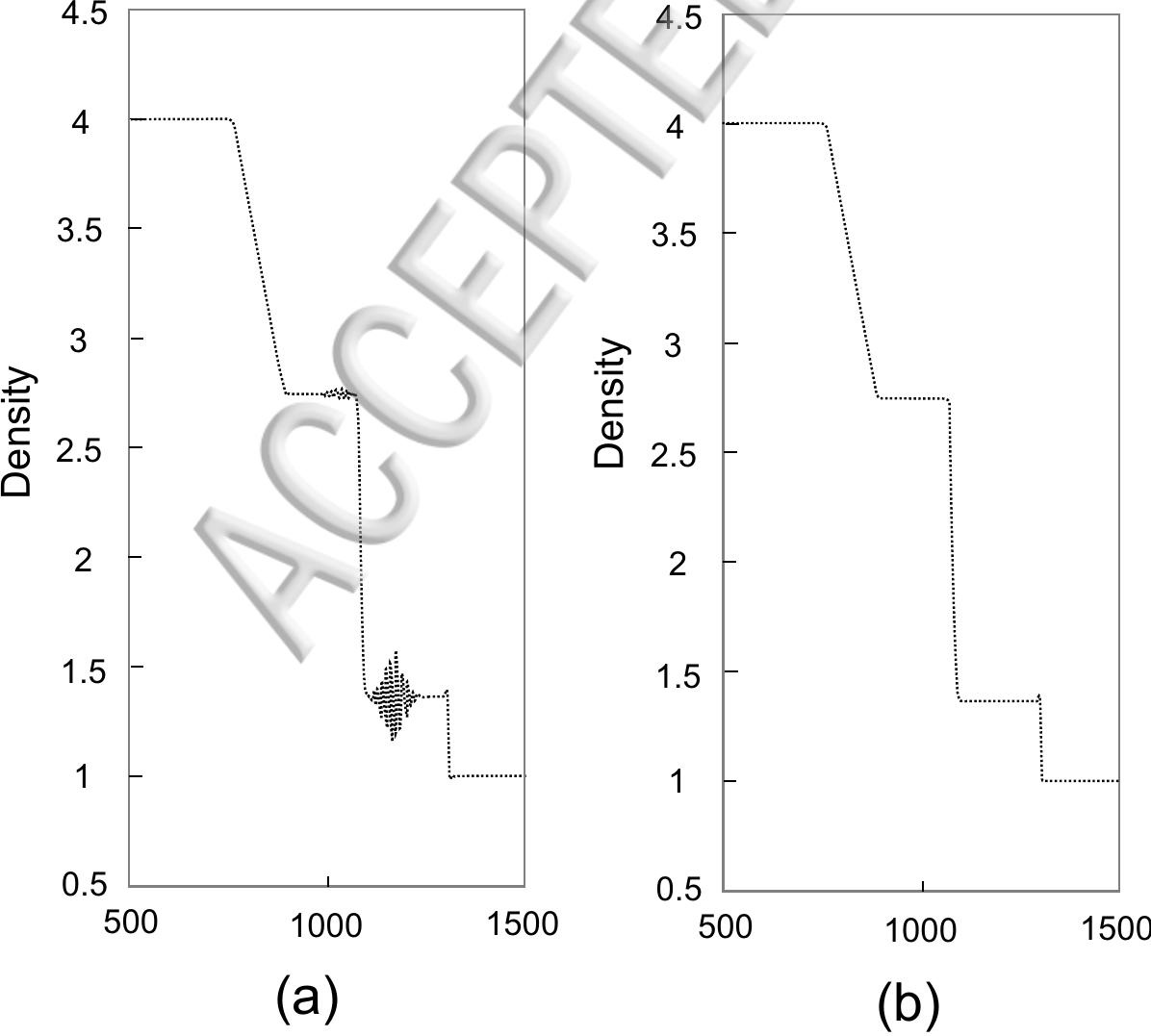


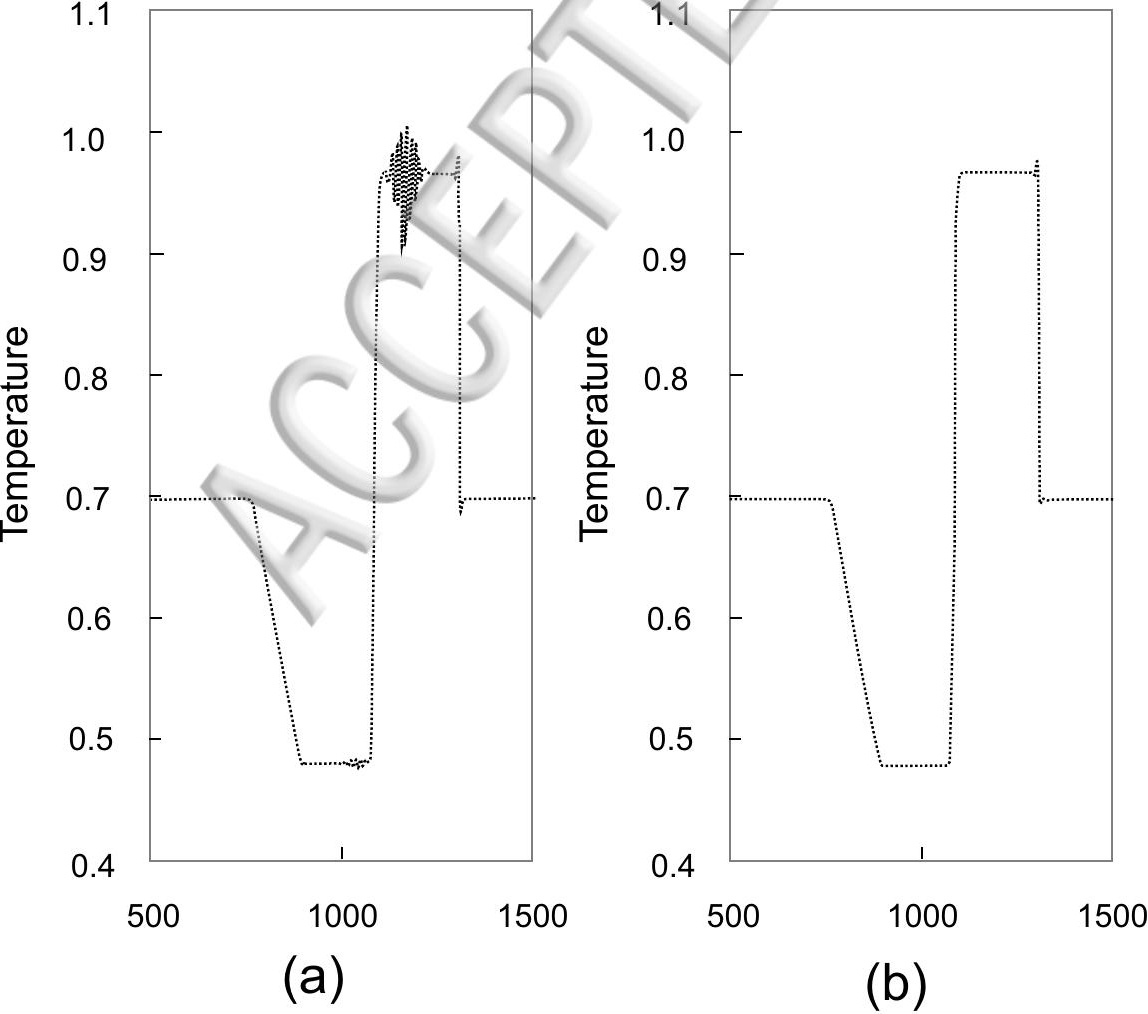




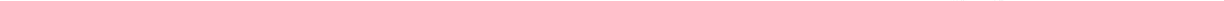

